\title{
Die Vegetationsentwicklung des Interglazials von Wallensen in der Hilsmulde
}

\author{
Von Ilse R a bi e n, Göttingen. Mit 11 Abb. \\ Aus dem Systematisch-Geobotanischen Institut der Universität Göttingen. \\ Einleitung. \\ I. Geographie, Klima, Geologie. \\ II. Stratigraphie der untersuchten Profile. \\ III. Die Arbeitsweise. \\ IV. Die Ergebnisse der pollenanalytischen Untersuchungen. \\ 1. Beschreibung der Diagramme. \\ 2. Die Gliederung der Waldentwicklung. \\ V. Die Knospenschuppenanalyse. \\ VI. Die übrigen Makrofossilien. \\ VII. Die Vegetationsentwicklung. \\ Zusammenfassung. \\ Literaturverzeichnis.
}

\section{Einleitung}

Seit etwa 1900 ist ein Vorkommen fossilführender interglazialer Schichten bei Wallensen in der Hilsmulde bekannt. Es liegt im Hangenden des Braunkohlentagebaus der Gewerkschaft Humboldt, $1 \mathrm{~km}$ südöstlich Wallensen (Bl. Salzhemmendorf). 1907 beschrieb Menzel diese Ablagerung als interglazial. Obwohl sie durch den immer weiter erfolgenden Abbau der Braunkohle wohl während der letzten 50 Jahre meist mehr oder weniger gut aufgeschlossen gewesen sein dürfte, wurde sie aber nicht weiter beachtet, bis Thомson (1947, vergl. 1951) eine erste pollenanalytische Untersuchung vornahm. Die Arbeit Thomson's bestätigte die Annahme Menzel's, daß es sich um ein Interglazial handele, und zwar um das letzte oder Saale-Weichsel-Interglazial. Da das Sediment äußerst fossilreich war, konnte man erwarten, daß eine gründliche Untersuchung der Makrofossilien - u. a. der bisher nur sehr wenig beachteten, hier sehr zahlreichen Knospenschuppen - sowie die Auszählung weiterer Pollendiagramme einen brauchbaren Beitrag zur Kenntnis der Vegetationsentwicklung des letzten Interglazials liefern würden. Es erschien auch wünschenswert, die letzten Reste des Interglazials, das offensichtlich zum größten Teil bereits abgebaut worden war, vor dem endgültigen Verschwinden zu bearbeiten.

In den Jahren 1948-50 hatte ich mehrmals Gelegenheit, in Wallensen Proben $\mathrm{zu}$ entnehmen. Ihre Untersuchung wurde Ostern 1949 begonnen und im August 1951 abgeschlossen.

Für die Anregung zu dieser Arbeit und mancherlei Rat möchte ich Herrn Prof. Dr. F. Firbas auch an dieser Stelle danken. Ferner danke ich ich den Herren Prof. Dr. H. Schmidt und Dr. G. Lüttig für geologische Hinweise, Frau G. Schneider und Herrn Dr. F. Koppe für Hilfe bei der Bestimmung der Knospenschuppen und Moose, Herrn Prof. Dr. P. W. Thomson für die Einsicht in unveröffentlichte Diagramme und Herrn Dr. H. Gross für die Überlassung einiger besonders fossilreicher Proben. 


\section{Geographie, Klima, Geologie}

Die Ith-Hils-Mulde gehört zum Weser-Leine-Bergland. Sie hat (RINK 1942) eine Längsausdehnung von $36 \mathrm{~km}$ und eine Breite bis $\mathrm{zu} 7 \mathrm{~km}$. Die Hilsberge erreichen Höhen bis $\mathrm{zu}$ etwa $480 \mathrm{~m}$. Die Grube Wallensen befindet sich im flacheren nordwestlichen Teil in dem Muldental der Saale, eines Nebenflusses der Leine, in einer Seehöhe von etwa $160 \mathrm{~m}$.

Klimatisch steht das Gebiet noch vorwiegend unter ozeanischem Einfluß, der allerdings im Beckeninnern schon ziemlich abgeschwächt sein dürfte. Nach HofFmeister, J. \& Schnelle, F. (1945) beträgt die mittlere Januartemperatur $0-0,5^{\circ}$, die mittlere Julitemperatur $17-18^{\circ}$, die mittlere Jahrestemperatur $8-8,5^{\circ}$, die mittlere jährliche Temperaturschwankung $16-16,5^{\circ}$. Die mittlere Niederschlagshöhe ist im Sommer (April-September) $420-480 \mathrm{~mm}$, im Winter (OktoberMärz) 360-420 mm, im Jahr $720-840 \mathrm{~mm}$. Die höchste Niederschlagsmenge wird im Juli, die geringste im März verzeichnet.

Die Hilsmulde wird von mesozoischen Gesteinen aus der Jura- und Kreidezeit aufgebaut. Das Braunkohlenbecken liegt in dem Einbruchsraum über dem Weenzer Salzstock (Fratschner 1950), dessen selbständige Bewegungen, begleitet von immer weiterem Absinken, offenbar bis ins Diluvium und Alluvium andauerten, wie die Ablagerungen über der Braunkohle beweisen.

Über dem Salzspiegel liegen ca. $100 \mathrm{~m}$ Tone, die vermutlich dem Neokom angehören, darüber Tone und Sande des Tertiärs, deren genaues Alter noch nicht bekannt ist. Im Beckentiefsten liegen über den Tonen und Sanden mächtige Braunkohlen und Braunkohlentone, die im unteren und mittleren Pliozän abgelagert worden sind (Thomson 1951). Die Braunkohlen sind (Fratschner 1950) leicht verfaltet und zeigen einen deutlichen Spezialsattel. Zahlreiche z. T. mehrere Meter mächtige Tonlinsen beweisen die unterschiedliche Senkungstendenz des Braunkohlenbeckens. Über der Braunkohle, deren Mächtigkeit ca. $100 \mathrm{~m}$ beträgt, folgen Gehängeschutt, dann das Interglazial (ca. $1 \mathrm{~m}$ mächtig), darüber wieder Gehängeschutt. In dem Gehängeschutt über und unter dem Interglazial befinden sich wenige umgelagerte $(\langle 1 \%)$ nordische Geschiebe, die aus glazialen Ablagerungen unsicheren Alters stammen, aber sicher älter als das Saale-Weichsel-Interglazial sind, wahrscheinlich noch älter als die Saale-Eiszeit. Schotter mit nordischen Geschieben stehen nordöstlich von Thüste an. Ihr Alter ist ebenfalls unsicher, entweder elster- oder saaleeiszeitlich. Zwischen Thüster und Duinger Berg befinden sich oberhalb des Steinbruches Marienhagen in beträchtlicher Höhe Schotter, die sicher aus der Elstereiszeit stammen (G. LǘtriG mdl.). Vereinzelte Vorkommen elstereiszeitlicher nordischer Geschiebe sind noch im Tal westlich Duinger Berg - Steinberg zwischen Duingen und Delligsen aufgeschlossen (G. LütTig mdl.). Geschiebemergel der Saalevereisung in normaler Ausbildung findet sich bei und nördlich von Hemmendorf.

Die Hilsmulde lag also im Randgebiet der Elster- und Saalevereisung. Von der letzten Vereisung, die die Elbe nicht überschritt, sind nur periglaziale Bildungen (Fließerde, Gehängeschutt) anzutreffen.

Menzel veröffentlichte 1907 folgendes Profil (von unten nach oben):

1. Jungtertiäre Braunkohlen: ca. $15 \mathrm{~m}$ mächtig aufgeschlossen.

2. „Grundmoräne“ mit Hilssandsteinschutt, Jurakalk und nordischem Material. (Unter „Grundmoräne“ verstand MEnzel allem Anschein nach das, was hier als Gehängeschutt bezeichnet wird.)

3. Fossilleere Mergelsande (kalkhaltige Feinsande).

7 Eiszeit und Gegenwart 
4. Bändertone mit wenigen Pflanzenresten, einigen Süßwasserkonchylien und Resten von Hecht und Barsch, wohl nur an der tiefsten Stelle des ehemaligen Sees.

5. Ziemlich kalkreiche Konchyliensande, reich an Süßwasserkonchylien, mit einigen Landschnecken und vorwiegend Sumpf- und Wasserpflanzen, u. a. viel Chara. Diese Sande sind nach Menzel höchstwahrscheinlich aus verwittertem Hilssandstein gebildet worden und sollen durch Zuflüsse in den See gelangt sein.

6. Sandige Torfe mit reicher Land- und Süßwassermolluskenfauna sowie Laubwaldflora.

7. Den Abschluß nach oben bildeten unreine, tonige, kalkfreie (alluviale) Torfe mit Säugetierresten, u. a. Elch und Edelhirsch,

8. darüber sandig-tonige Bildungen.

Die Interglazialschichten (4-6) waren nach Menzel besonders entlang einer rordwestlich streichenden Verwerfung gestört und zum Teil „aufgerichtet, gefaltet und verworfen“. Darüber lagerte diskordant das Alluvium (7, 8).

Thomson (1951) stellte auf Grund pollenanalytischer Untersuchungen fest, daß die Braunkohle pliozänes, frühestens obermiozänes Alter hat. Das Interglazial wurde von ihm dem Saale-Weichselinterglazial (Eem-Interglazial) zugeordnet, nachdem schon MENzeL die unter dem Interglazial liegenden moränenartigen A.blagerungen der Hauptvereisung (Saalevereisung) zugewiesen hatte. Heute ist durch den fortgesetzten Abbau der Braunkohle kein unmittelbarer Zusammenhang zwischen den von Menzel beschriebenen und den von mir untersuchten Schichten mehr festzustellen. Jedoch dürfte die heute noch vorhandene Grobdetritusgyttja mit den von MEnzel beschriebenen „sandigen, blätterreichen Torfen" identisch sein. Konchyliensande und sonstige tierische Fossilien wurden bei der jetzigen Untersuchung nicht gefunden. Die heute im Ostteil der Grube aufgeschlossene See- und Moorablagerung mit der Allerödtuffschicht (vergl. Firbas 1951) ist vielleicht mit den unreinen Torfen Menzel's identisch.

Die interglaziale Ablagerung muß ursprünglich erheblich mächtiger gewesen sein. Es wurden Zapfen von Picea abies gefunden, die auf $1 / 5$ ihrer ursprünglichen Dicke zusammengedrückt worden waren. Dicke Stämme, die in dem ufernahen Schwemmtorf vorkamen, waren auf weniger als $1 / 3$ ihres ursprünglichen Durchmessers zusammengepreßt.

Der Interglazialsee von Wallensen muß annähernd die gleiche Ausdehnung gehabt haben wie der heutige Braunkohlentagebau. Vor 50 Jahren, als der Abbau der Braunkohle noch lange nicht so weit vorgetrieben war wie heute, war das Interglazial schon aufgeschlossen, und auch heute sind noch die letzten Reste davon zu sehen.

\section{II: Stratigraphie der untersuchten Profile}

Im Nordostteil der Grube wurden 1948 und 1949 mehrere Profile untersucht.

Profil I stammt von einer abgerutschten Scholle, die sich wenige Meter über der Braunkohle befand (Tiefenangabe von der Oberkante der untersuchten Schicht nach unten).

Hangendes: Hilssandsteinschutt.

$0-10 \mathrm{~cm}$ : Homogene schwarzbraune Mudde mit dünnen $Z$ wischenlagen von hellem Sand. 
$10-20 \mathrm{~cm}$ : Wie vorher, unten blättrig.

$20-45 \mathrm{~cm}$ : Blätterreiche Schicht („Blätterschicht“).

$45-50 \mathrm{~cm}$ : Mehr muddeartig, weniger aufblätternd.

$50-53 \mathrm{~cm}$ : Hellgrauer Sand.

$53-58 \mathrm{~cm}$ : Tonige Mudde.

$58-85 \mathrm{~cm}$ : Tonige Mudde mit aufgearbeiteten Braunkohlenbrocken.

Darunter geschichteter lehmiger Feinsand mit immer größer werdenden Braunkohlenbrocken und gelblichbraunen verwitterten Sandsteinstückchen.

P r of il I a (vergl. Abb. 1) befand sich ca. $20 \mathrm{~cm}$ östlich von I. Die Schichtenfolge entspricht genau dem Profil I. Es diente zur Pollenanalyse.

Pr of i I I (Abb. 2) lag $3 \mathrm{~m}$ westlich von I und Ia. Die Proben wurden von der Oberkante der schwarzbraunen Mudde zum Hangenden hin entnommen.

$120-70 \mathrm{~cm}$ : Graue tonige Schichten von Bruchteilen von $1 / 10$ bis mehrere $\mathrm{mm}$, wechsellagernd mit weißlichem Sand von gleich wechselnder Stärke.

$70-65 \mathrm{~cm}$ : Stark humoser schwarzbrauner Ton, fast ohne Sand.

$65-.0 \mathrm{~cm}$ : Schutt aus sehr schwach gerundeten Sandsteingeröllen, z. T. noch ziemlich tonig mit sandigem Zwischenmittel.

$0-10 \mathrm{~cm}$ : Schwarzbraune Mudde.

Profil II I stammt ebenfalls von einer abgerutschten Scholle etwa $30 \mathrm{~m}$ östlich von I und II und etwa $10 \mathrm{~m}$ höher. Diese Scholle war von ihrem ursprünglichen Standort noch nicht weit entfernt.

$0-15 \mathrm{~cm}$ : Grauer Ton mit hellen sandigen Zwischenlagen.

$15-35 \mathrm{~cm}$ : Gut spaltende muddeartige schwarzbraune Schichten, der Blätterschicht entsprechend.

$35-50 \mathrm{~cm}$ : Kompaktere dunkelgraubraune Schichten, stellenweise mit moosreichen Zwischenschichten.

$50-60 \mathrm{~cm}$ : Schwarzbrauner Ton mit hellen Zwischenschichten.

60-100 cm: Dunkelgrauer Lehm mit Kohlebröckchen und helleren Gesteinsstückchen.

Das Profil III befand sich etwa $12 \mathrm{~m}$ unter der Oberkante der Grubenwand.

Ein weiteres Profil wurde einige Meter nördlich von III an der ungestörten Grubenwand gewonnen, etwa $10-11 \mathrm{~m}$ unter ihrer Oberkante. Unter der Blätterschicht befand sich eine ca. $5-10 \mathrm{~cm}$ mächtige helle Sandschicht mit Hilssandsteingeröllen, darunter schwarzer, sandiger Ton mit kleinen Sandsteingeröllen und kohliger Substanz. Die Liegendgrenze war nicht aufgeschlossen. Die $40-45 \mathrm{~cm}$ mächtige Blätterschicht war, wie die spätere Untersuchung ergab, in sich gestört, offenbar durch die Ufernähe und eine dadurch bedingte häufige Umlagerung (TномsоN weist darauf hin, daß an den Ostufern von Seen in Gebieten mit vorherrschenden Westwinden häufig derartige Umlagerungen stattfinden). Die Blätterschicht fiel ca. $20-30^{\circ}$ nach Westen ein. Das Hangende der Blätterschicht war ein sandiger Ton mit reinen Tonlagen, übergehend in gelbbraunen Sand mit Hilssandsteingeröllen. Die Proben dieses Profils wurden als Einzelproben behandelt.

Außerdem wurden noch Proben untersucht, die bereits 1947 von Herrn Dr. F. Gross (damals am Amt für Bodenforschung in Hannover) gesammelt und Herrn Prof. Firbas freundlicherweise zur Verfügung gestellt worden waren. Schließlich wurden noch zahlreiche nicht orientierte Stücke, die besonders gutes Fossilmaterial versprachen, zur Untersuchung mitgenommen. Das Alter dieser Proben ist pollenanalytisch bestimmt worden.

Insgesamt wurden so 3 Profile auf ihren Pollengehalt sowie eines davon und etwa 50 pollenanalytisch eindatierte Einzelproben auf ihre Großreste untersucht. 


\section{Die Arbeitsweise}

P olle nan a y s : Die zur Pollenanalyse bestimmten Proben wurden nach 3 verschiedenen Verfahren behandelt.

a) Ein Teil der Proben wurde nach einer Angabe von Herrn Prof. Thomson mit $\mathrm{H}_{2} \mathrm{O}_{2}$ behandelt. Die Stücke wurden zerkleinert, dann ca. 5 Minuten in $10 \%$ igem $\mathrm{H}_{2} \mathrm{O}_{2}$ erhitzt bzw. gekocht und durch ein feines Sieb gegossen. Danach wurden sie ausgewaschen und noch einige Minuten mit kalter $10 \%$ iger KOH behandelt, hierauf wieder ausgewaschen und in Glyzerin aufbewahrt. Es wurde darauf geachtet, daß die verarbeitete Materialmenge möglichst gleich groß war und daß die Proben in möglichst der gleichen Menge Glyzerin aufbewahrt wurcien.

b) Ein anderer Teil der Proben wurde nach der üblichen Methode mit 10\%iger $\mathrm{KOH}$ einige Minuten gekocht.

c) Einige sehr tonhaltige Proben wurden nach vorheriger Behandlung mit $\mathrm{KOH}$ oder $\mathrm{H}_{2} \mathrm{O}_{2}$ mit $\mathrm{HF}$ aufgeschlossen.

Es stellte sich heraus, daß bei der Behandlung mit $\mathrm{H}_{2} \mathrm{O}_{2}$ die Proben am besten aufgeschlossen wurden. Jedoch hatte diese Methode den Nachteil, daß die einzelnen Pollenkörner so stark aufgehellt wurden, daß sie mit Safraninrot angefärbt werden mußten, was wieder die Deutlichkeit in Bezug auf die Strukturen der Pollenkörner beeinträchtigte.

Bei der Behandlung mit $\mathrm{KOH}$ wurden die Proben zwar weniger gut aufgeschlossen, die Pollenkörner waren aber deutlicher zu erkennen, da sie nicht so stark aufgehellt worden waren.

Beim Auszählen der Proben wurde nach der v. Post'schen Methode vorgegangen. Die einzelnen Proben wurden jeweils bis auf 200 Baumpollen ausgezählt, bei sehr pollenarmen oder besonders haselreichen Proben wurden weniger Baumpollen (100 bzw. 50) ausgezählt. Die Haselpollen wurden nicht in die Baumpollensumme mit einbezogen. Auch Acer und Salix wurden ihres seltenen Vorkommens wegen außerhalb der Baumpollensumme gezählt. Die Baumpollendichte wurde auf Baumpollen je $\mathrm{cm}^{2}$ bestimmt. Die Proben wurden bei etwa 500 facher Vergrößerung ausgezählt.

Großrestanalyse: Die zur Untersuchung auf Großreste bestimmten Proben wurden während einiger Wochen mit $15 \%$ iger $\mathrm{HNO}_{3}$ behandelt und dann in der gleichen Lösung so lange gekocht, bis sie zerfielen. Vor dem Kochen wurden sie noch vorsichtig gespalten, um besonders gut erhaltene Blattreste zu retten. Nach dem Kochen wurde die ganze Masse in ein feines Sieb getan, und die feinen Bestandteile wurden vorsichtig abgeschlämmt.

IV. Die Ergebnissederpolienanalytischen Untersuchungen

1. Beschreibung der Diagramme

Thomson hat 1951 bereits 2 Diagramme des Interglazials von Wallensen veröffentlicht (im folgenden als TH. „I" und „III“ bezeichnet). Die Proben stammen ebenfalls von der Nordostwand des Aufschlusses und wurden 1946 und 1947 entnommen. Thомsом unterscheidet hierbei liegende autochthone Schichten (Detritusgyttja und z. T. ufernaher Schwemmtorf mit viel Osmunda), darüber allochthone Ablagerungen aus Sand, Schluff, Ton, Torf und Hilssandstein, die offenbar unter Zerstörung von Hochmooren (viel Ericaceenpollen und Sphagnumsporen, Reste von Eriophorum vaginatum) am Ende des Interglazials im arktischen Klima entstanden sind. Sie enthalten besonders im oberen Teil viel älteren interglazialen und tertiären Pollen auf sekundärer Lagerstätte. 


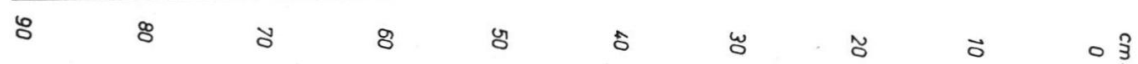

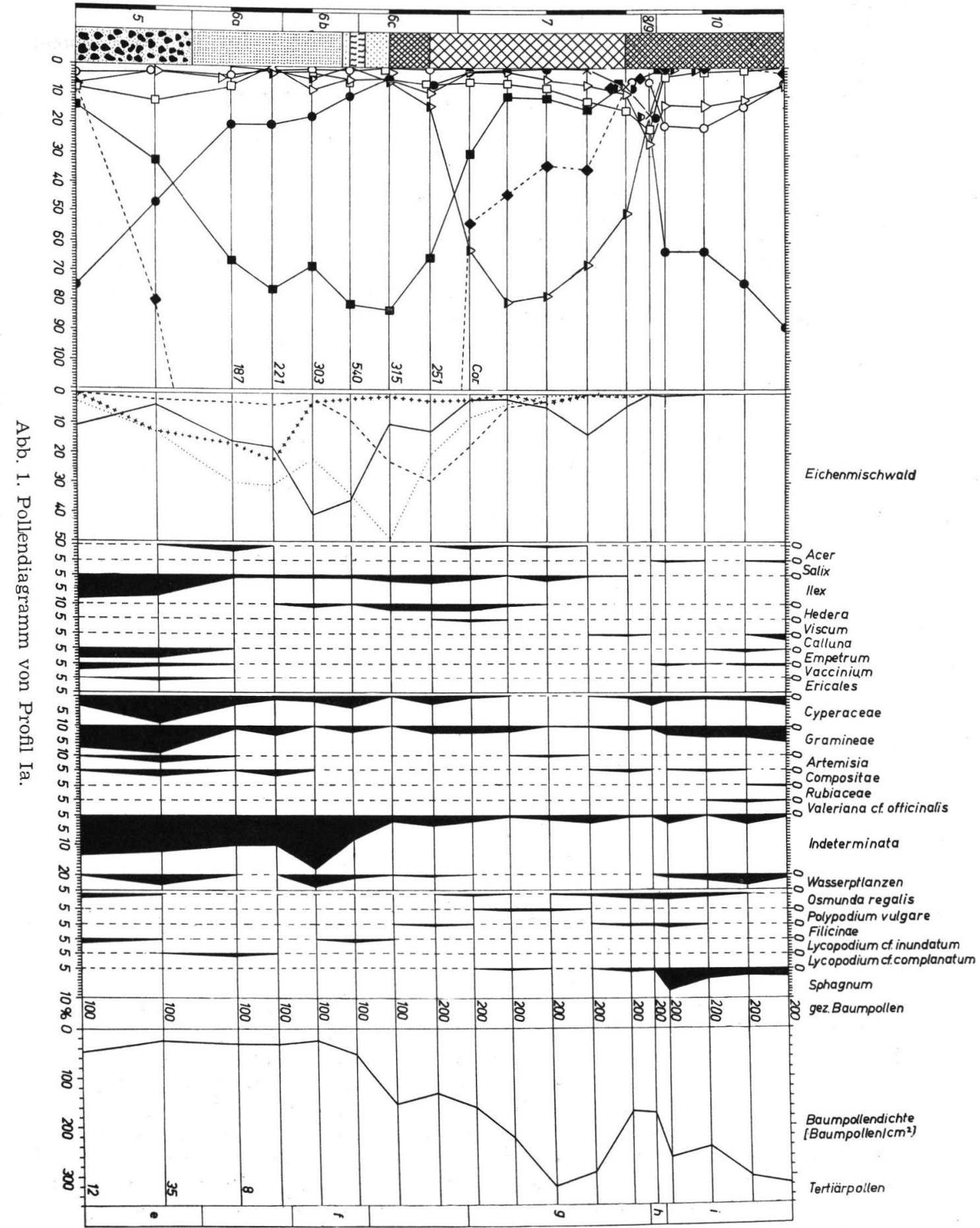


Meine eigenen Profile, die infolge des fortschreitenden Abbaus nicht mehr so günstig lagen, bestätigen und ergänzen z. T. die Befunde Tномson's.

Die Abfolge der Waldperioden stimmt, soweit nachgewiesen, mit jener überein, die für das letzte Interglazial aus Dänemark und verschiedenen Teilen des norddeutschen Flachlandes schon seit langem bekannt ist (Jessen \& Milthers 1928, Woldstedt 1949, Woldstedt, Rein \& Selle 1951). Mit Thomson darf man hierin den Beweis erblicken, daß die Ablagerung von Wallensen in der Riß-Würm(Saale-Weichsel-, Eem-)Interglazialzeit gebildet worden ist.

Profil I a (Abb. 1): Der Anfang des Diagramms fällt in eine ausklingende Kiefern- und beginnende Hasel-Eichenmischwaldzeit; die Kiefer hat zunächst noch einen Wert von 75\%. Zu Beginn der Hasel-Eichenmischwaldzeit dominiert die Ulme mit $31 \%$, auch die Esche hat erstaunlich hohe Werte $\left(23^{\circ} \%\right)$. Esche und Ulme treten dann zurück, und es erfolgt der Anstieg der Eiche, die mit $41 \%$ ihren Höchstwert erreicht. Gleichzeitig steigt die Hasel weiter an, ihr Maximalwert beträgt 540\% (zur gleichen Zeit mit dem Eichenmischwaldmaximum). Mit dem Rückgang der Eiche steigt die Ulme ein zweites Mal an (bis 49\%) und mit ihr die Linde, die bisher nur mit wenigen \% vertreten war. Zur Zeit des Lindenmaximums $(29,5 \%)$ ist die Ulme wieder stark im Abfall begriffen, die Hasel hat noch Werte über 250\%. Mit dem Rückgang des Eichenmischwaldes und der Hasel findet eine starke Ausbreitung der Hainbuche und zur gleichen Zeit ein schwacher Anstieg der Erlenkurve statt. Während der Hainbuchenzeit gehen die Eichenmischwald- und Haselwerte immer mehr zurück; gegen Ende der Hainbuchenzeit sind diese Holzarten nur noch mit Werten wenig über $0 \%$ vertreten. Die Hainbuche hat ein Maximum von $80 \%$.

Der Anstieg von Tanne und Fichte beginnt zu der Zeit, da die Hainbuche roch durch relativ hohe Werte vertreten, aber schon im Rückgang begriffen ist. Die Tanne tritt hier erstmalig auf, während die Fichte schon durch das ganze Diagramm mit Werten unter 10\% vorhanden war. Die Gipfel beider Arten fallen zusammen (Tanne: 16\%, Fichte: 26\%). Die Erle erreicht gleichzeitig mit Tanne und Fichte ihren höchsten Wert $(20 \%)$, fällt dann aber schnell wieder zu wenigen $\%$ ab. Während des Abfalls der Fichte beginnt ein schwacher Anstieg der Kiefer. Während die Kiefernwerte weiter zunehmen, gehen die Birke wieder und die Fichte weiter zurück. Damit endet das Diagramm. Es erweitert die Befunde Thomson's durch den Nachweis einer kiefernreichen Periode vor der Eichenmischwaldzeit und die ziemlich hohen Werte von Fraxinus. Die schwarzbraune Mudde mit sandigen Zwischenlagen im Hangenden deutet möglicherweise den Beginn der allochthonen Serie Thomson's an, doch wurden hier keine Tertiärpollen gefunden. Solche konnten nur zu Beginn der Eichenmischwaldzeit in den drei untersten Proben reichlich festgestellt werden.

Profil III: Das hier nicht wiedergegebene Diagramm ist weniger vollständig als Ia, jedoch läßt sich auch hier eine sehr ähnliche Entwicklung erkennen. Es beginnt später als Ia. Die Hasel ist schon gleich mit Werten von über $300 \%$ vertreten, sie erreicht hier ein Maximum von über 900\%. Die Gipfel der Kurven der einzelnen Komponenten des Eichenmischwaldes sind etwas mehr verwischt; es läßt sich keine zeitliche Aufeinanderfolge mit Sicherheit feststellen. Ebenso sind die Gipfel der Tannen- und Fichtenkurve nicht erfaßt worden. In den obersten Schichten läßt sich ein starker Anstieg der Ericaceen- und Sphagnumwerte erkennen, der auf eine Vermoorung hinweist. Tertiäre Pollen wurden fast nur in den beiden untersten und in der obersten Probe gefunden.

Profil II (Abb.2): Es steht offenbar zu den „allochthonen“ Abschnitten der Tномson'schen Diagramme in Beziehung. In den beiden untersten Proben, 


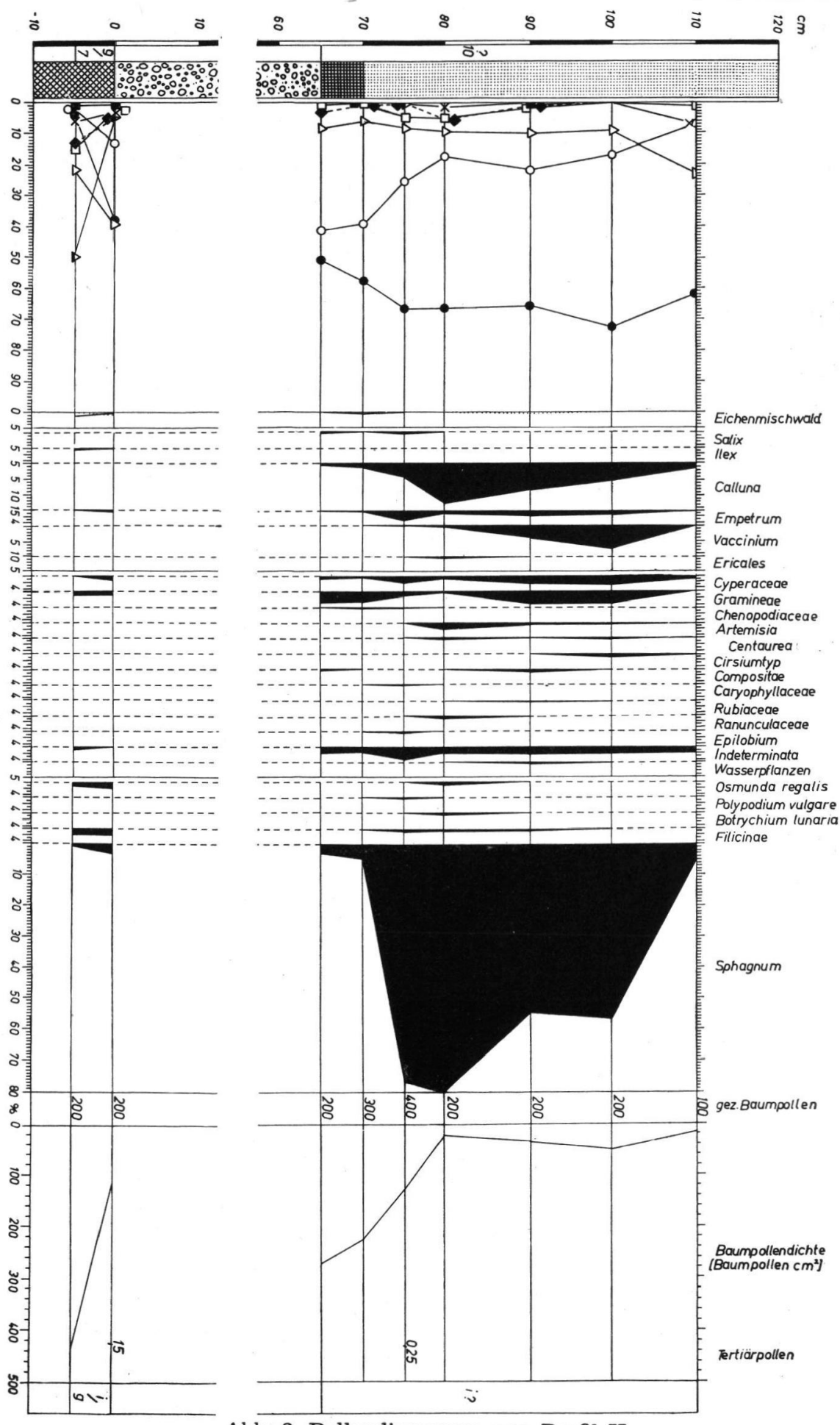

Abb. 2. Pollendiagramm von Profil II. 


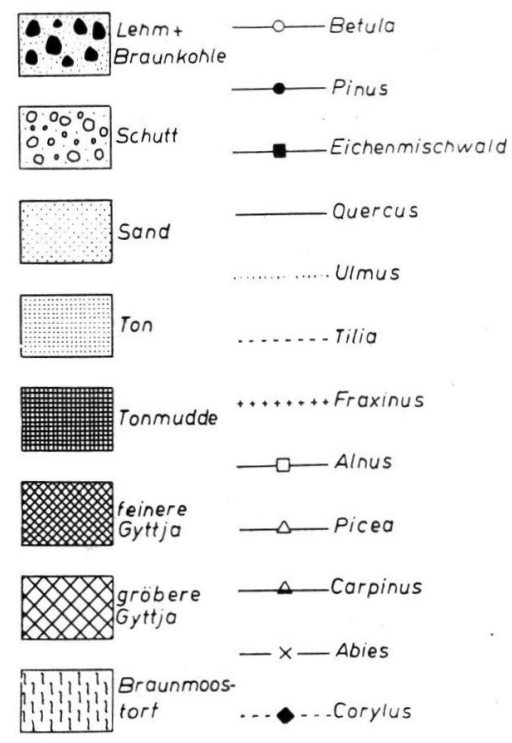

Zeichenerklärungen zu den Abb. 1 und 2. die noch zu den „autochthonen“ Schichten des Interglazials gehören, sehen wir ein Abfallen der Hasel-, Eichenmischwald-, Hainbuchenund Tannenkurve und ein Ansteigen der Fichten-, Kiefern- und Birkenkurve. Es dürfte sich hier etwa um den Beginn der Fichtenzeit handeln. Darüber folgen $65 \mathrm{~cm}$ Schutt aus Hilssandsteingeröllen mit tonigsandigem Zwischenmittel. Diese Schichten wurden nicht untersucht; es handelt sich hierbei, ebenso wie bei dem darüberliegenden mehr tonigen Material, sehr wahrscheinlich um periglaziale Bildungen (Fließerde usw.). Erst aus den darüber liegenden stärker tonigen Schichten wurden wieder Proben untersucht. Hier zeigt sich sogleich ein starker Anstieg der Hochmoorelemente - Ericaceen und Sphagnum -; ferner sind Kiefer und Birke stark vertreten, die Kiefer von $50 \%$ ansteigend auf über $70^{\circ} \%$ bei $100 \mathrm{~cm}$ (bei $110 \mathrm{~cm}$ immer noch über $60 \%$ ), die Birke von $41 \%$ abfallend auf $7 \%$ bei $110 \mathrm{~cm}$.

Die Fichte steigt von Werten zwischen 5 und $10 \%$ auf $23 \%$ bei $110 \mathrm{~cm}$ an. Eichenmischwald, Hasel, Hainbuche, Erle und Tanne sind nur spärlich vertreten, die Tanne in der obersten Probe mit 7\%. Dieser spärliche Pollen könnte sich auf sekundärer Lagerstätte befinden. Die Baumpollendichte fällt von über 400 bis $500 / \mathrm{cm}^{2}$ in den unteren auf unter $50 / \mathrm{cm}^{2}$ in den obersten Proben. Einwandfrei tertiäre Pollenkörner konnten nur ganz wenige bestimmt werden; vielleicht gehört dazu noch ein Teil der sehr schlecht erhaltenen unbestimmbaren Pollen, die sich um etwa $5 \%$ bewegen und nur einmal $16 \%$ erreichen. Der Pollenführung nach besteht zwischen dem allochthonen Abschnitt Tномson's und dem Profil II eine ziemlich gute Utbereinstimmung mit dem Unterschied, daß die Tertiärpollen in II schwächer vertreten sind. Ob die Kiefernzeit des Profils II einfach eine Fortsetzung der Kiefernzeit von Profil Ia ist, kann nicht entschieden werden. Auf Grund der liegenden Schuttmassen wäre es auch möglich, daß der Kiefernzeit von Profil II bereits eine Zeit noch ungünstigerer Klimaverhältnisse vorausging. Es handèlt sich also vielleicht um ein frühwürmglaziales interstadial (vgl. SELle 1952). Der Abbau der Schichten verhinderte eine weitere Untersuchung der Frage.

\section{Die Gliederung der Waldentwicklung}

Nach den 5 vorliegenden Diagrammen läßt sich nun die Waldentwicklung folgendermaßen gliedern (arabische Zahlen) und mit der dänischen Gliederung von Jessen \& Milthers (kleine Buchstaben) und der nordwestdeutschen Gliederung von Selle (in Woldstedt, Rein \& Selle 1951; römische Zahlen) vergleichen.

Kiefern-Eichenmischwaldzeit: Pinus vorherrschend, Corylus und die Bäume des Eichenmischwaldes in kräftiger Ausbreitung begriffen. Picea, Alnus und Betula mit geringen Werten vorhanden.

Grenze: Pinus $\times$ Eichenmischwald. 
$6(\mathrm{f}, \mathrm{VI})$

b̉a (VIa)

$6 \mathrm{~b}(\mathrm{VIb})$

6c (VIc)

i (g, VIIa)

8 (g/h, VIIb?) : Tannenzeit: Bei gleichzeitigem Rückgang von Carpinus und Anstieg von Picea erreicht Abies ein Maximum $(46 \%)$. Alnus ist ziemlich häufig $(21 \%)$.

Grenze: Picea $\times$ Pinus.

$y$ (h, VIII, IX) : Fichtenzeit: Picea vorherrschend, Carpinus und Eichenmischwald auf Werte unter $10 \%$ abgesunken. Pinus gegen Ende stark ansteigend.

Grenze: Pinus $\times$ Picea.

$10(\mathrm{i}, \mathrm{X})$
Hase 1-Eichenmischwaldzeit: Corylus fast durchweg häufiger als die Baumpollensumme. Nächstdem der Eichenmischwald vorherrschend. Weiter zu gliedern in:

Ulmus (bis $46^{\circ} \%$ ) und Fraxinus (bis $23^{\circ} \%$ ) und noch reichlichem Auftreten von Pinus, mit wenig Tilia und noch ohne Carpinus. lus- und Quercus-Maximum (915 bzw. 62\%). Mit viel Ulmus, beginnender Ausbreitung von Tilia und mit etwas Carpinus. Corylus-Abfall und der Ausbreitung von Carpinus.

Grenze: Eichenmischwald $\times$ Carpinus. $80 \%$ ), Pinus und Betula sehr spärlich, in einzelnen Proben fast fehlend. Picea und Alnus in geringer Zunahme; gegen Ende Auftreten und erste Ausbreitung von Abies.

Kiefernzeit: Pinus herrscht vor, nächstdem sind Betula und Picea häufig. Pollen anspruchsvoller Arten fehlt oder ist nur mit Werten unter 10\% vorhanden (Abies, Alnus, Carpinus, Eichenmischwald). Unter den Nichtbaumpollen viel Ericales (besonders Calluna), außerdem viel Sphagnum.

Vielleicht in einen älteren birkenreicheren (10a) und in einen jüngeren birkenärmeren (10b) Abschnitt zu gliedern.

\section{Die Knospenschuppenanalyse}

Die große Menge von Knospenschuppen in den Proben legte neben ihrer Bestimmung auch eine statistische Auswertung nahe. Die Beschreibung der einzelnen Gattungen bzw. Arten, die bestimmt werden konnten, wird an anderer Stelle erfolgen (Paläontologische Zeitschrift). Hier soll nur auf die statistische Auswertung eingegangen werden.

Hesmer hat 1935 in einer Arbeit über Samen- und Knospenschuppenanalysen in postglazialen Mooren der Mark Brandenburg erstmals Diagramme von Knospenschuppen veröffentlicht und gezeigt, daß die quantitative Knospenschuppenanalyse eine wichtige Ergänzung zur Pollenanalyse und zu den Untersuchungen von sonstigen Großresten darstellt. In Abb. 3 ist nun das zu Profil I bzw. Ia (vergl. Abb. 1) gehörige Knospenschuppendiagramm dargestellt, wobei die Waldperioden links hinzugefügt sind. Für das Diagramm wurde die logarithmische Form der Darstellung gewählt und zwar, da $\log 1=0$, wurde für sämtliche Werte der $\log \mathrm{x}+1$ aufgetragen.

Die Eiche, die wegen der großen Schuppenzahl der Knospen und ihrer guten Erhaltungsfähigkeit sicher übervertreten ist, ist schon fast von der untersten 
Probe an vorhanden. Ihre Höchstwerte liegen einmal in der Hasel-Eichenmischwaldzeit im Abschnitt 6a/b und dann in den Abschnitten 6c-7, also zur Zeit des Hainbuchenanstiegs und -Maximums und des Eichenmischwaldabfalls. Obwohl die Eiche im Pollengehalt um diese Zeit 10\% kaum überschreitet, muß sie in der Nähe noch sehr häufig gewesen sein. Jedenfalls sind die Funde ein schöner Beweis dafür, daß die Eiche tatsächlich vom Beginn der Eichenmischwaldzeit bis zum Ende der Hainbuchenzeit in Wallensen vorhanden war.

Die Zitterpappel trägt an der einzelnen Knospe nur etwa halb soviel Schuppen wie die Eiche. Sie zeigt 2 Maxima, nämlich während des Eichenmischwaldanstiegs $(6 a / b)$ und während der Hainbuchenzeit (7). Während der Lindenzeit ist sie nur sporadisch anzutreffen.

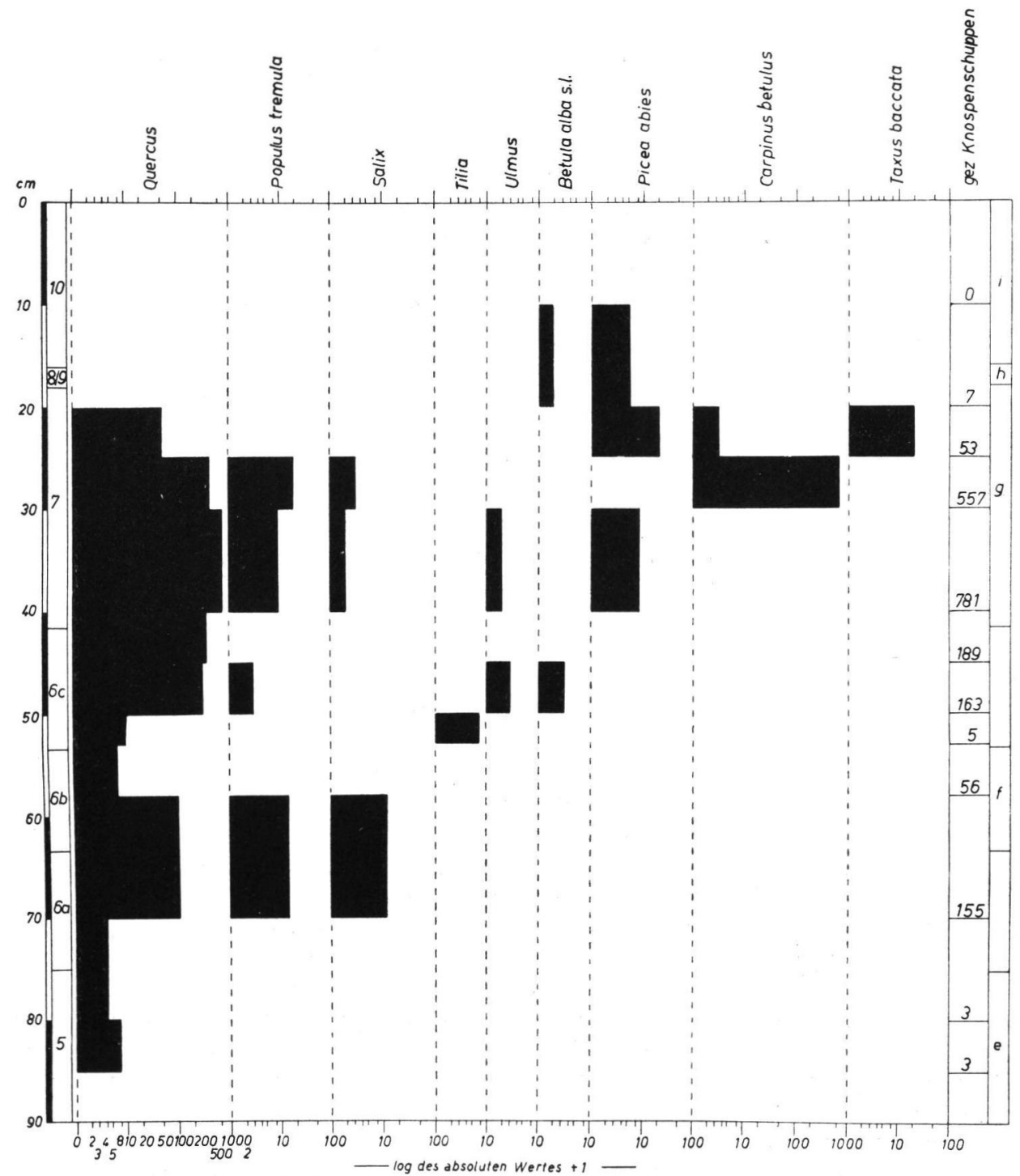

Abb. 3. Knospenschuppendiagramm von Profil Ia. 
Die Häufigkeit der Weidenschuppen entspricht weitgehend dem Verhalten der Zitterpappel. Die Linde ist nur mit wenigen Schuppen aus der Zeit des lindenanstiegs vertreten. Besonders schön ist der Fund von Ulmenschuppen, da sonst keine Makrofossilien von Ulmus gefunden worden sind. Sie stammen aus dem Ende der Hasel-Eichenmischwaldzeit (6c) und der älteren Hälfte der Hainbuchenzeit (7), wo die Pollenwerte für Ulmus schon unter 10\% liegen.

Die Schuppen der Birke zeigen, daß nicht nur in den letzten Abschnitten des Interglazials, in denen hohe Birkenpollenwerte verzeichnet sind, Birken im Wallenser Walde wuchsen, sondern auch in der Eichenmischwaldzeit (6c) vereinzelte Bäume dort vorhanden waren, obwohl die Pollenhäufigkeit unter $1 \%$ liegt.

Die Fichte ist durch Schuppen von der Hainbuchenzeit bis in die Kiefernzeit vertreten, ihre Höchstwerte liegen in der Fichtenzeit. Auch zu der Zeit, da der Anstieg der Fichtenkurve mit Werten unter 5\% beginnt, wurden einige Fichtenschuppen gefunden, die Fichte war also zur Zeit dieser geringen Pollenwerte schon in der Umgebung des Sees vorhanden. Schuppen der Hainbuche wurden nur im jüngeren Teil der Hainbuchenzeit, in diesem aber reichlich nachgewiesen.

Eibenschuppen traten nur in einer einzigen Probe aus dem Ende der Hainkuchenzeit auf, obwohl die Eibe durch andere Reste erheblich lange, vom Eichenmischwaldmaximum bis zum Ende der Hainbuchenzeit, in Wallensen nachgewiesen worden ist. Das liegt wohl an der außerordentlichen Zartheit der Eibenschuppen. Ihr Vorkommen scheint von sehr günstigen Erhaltungsbedingungen abhängig zu sein. Anscheinend sind sie sonst noch nirgends nachgewiesen worden.

Knospenschuppen der hier erwähnten Arten wurden auch in zahlreichen Einzelproben in \pm großer Menge gefunden. Sie sollen im nächsten Kapitel mit aufgeführt werden.

Besäße man nur das Knospenschuppendiagramm, so kämen zwar einige Züge der interglazialen Waldentwicklung richtig heraus, aber wesentliche Teile blieben unbekannt (vergl. z. B. das Fehlen von Corylus, Abies und Pinus unter den Schuppen). Aus einem Vergleich des Knospenschuppendiagramms mit dem Pollendiagramm geht also hervor, daß man die Knospenschuppenanalyse zwar als gute Ergänzung zur Pollenanalyse verwer.den kann (Populus, Taxus), daß man aber die Funde mit sehr großer Vorsicht deuten muß und sich auf keinen Fall verleiten lassen darf, aus hohen Werten nun auf die Vorherrschaft einer bestimmten Baumart oder aus dem Fefılen von Schuppen auf ihr tatsächliches Fehlen zu schließen.

Wertvoll sind die Funde von Schuppen in Proben, in denen der Pollen der betreffenden Art oder Gattung nur wenige \%, z. T. unter 1\%, ausmacht und daher das Vorkommen der Art im Gebiet nicht belegt. Es wäre wertvoll, einmal Versuche an einzelstehenden Bäumen zu machen, wieweit die Knospenschuppen vertragen werden, oder Oberflächenproben in reinen Beständen und in Mischwäldern auf ihre Zusammensetzung an Knospenschuppen zu untersuchen.

\section{Die übrigen Makrofossilien ${ }^{1}$ )}

1. Coenococcum geophilum: Etwa 200 Sklerotien. In 8 selten, $8 / 9$ und 9 häufig, 9/10 sehr häufig. Besonders in an organischem Material reichen Schwemm-

1) Bei den Angaben über die Häufigkeit bedeuten: sehr selten $\mathbf{1}-\mathbf{5}$, selten $\mathbf{5}-\mathbf{2 0}$, häufig 20-100, sehr häufig über 100 Stück. Die arabischen Zahlen geben die Waldperioden an (vergl. S. 104), z. T. sind mit römischen Zahlen auch die Profile erwähnt. Alle Funde wurden morphologisch und anatomisch eingehend untersucht und verglichen. Eine nähere Beschreibung wird hier aber nur bei seltenen und schwierig zu bestimmenden Arten gegeben. 
torfproben, zusammen mit viel Coniferenresten. Nach C. A. WEber (1914) ist Coenococcum geophilum in aus terrestrischem Humus gebildeten oder solchen enthaltenden Böden von Heiden und Wäldern Europas weit verbreitet.

2. Sphagnum sp.: Sporen vereinzelt in Ia 7, III 6 a/b $(-10 \%)$. Anstieg in 7, 8, 9 und 10 (in Ia nur schwach ausgeprägt, in III starker Anstieg bis über $50 \%$. In II 10? bis zu 80\%). In den beiden Thomson'schen Diagrammen im Abschnitt 10 ebenfalls starker Anstieg der Ericaceen- und Sphagnumkurve.

Nicht näher bestimmbare Blattreste wurden vereinzelt in den Abschnitten 9 und 10 sefunden. Tномson erwähnt aus seinem allochthonen Teil ebenfalls viele Blattreste.

3. Aulacomnium palustre Schwgr.: Etwa 10 Stämmchen. In $6 \mathrm{a} / \mathrm{b}$ selten, in 10 sehr selten.

4. Leucodon sciuroides L.: In $6 \mathrm{a} / \mathrm{b}, 6 \mathrm{~b} / \mathrm{c}$ und $6 \mathrm{c}$ sehr selten.

5. Neckera complanata Hüb.: In $6 \mathrm{a} / \mathrm{b}, 6 \mathrm{~b} / \mathrm{c}$ und $6 \mathrm{c}$ sehr selten, in 7 selten.

6. Fontinalis antipyretica L.: In 9 sehr selten, 10 selten.

7. Drepanocladus fluitans Warnst.: In $6 \mathrm{a} / \mathrm{b}, 6 \mathrm{~b}, 6 \mathrm{~b} / \mathrm{c}$ und $6 \mathrm{c}$ häufig, in $7,8,9$ und 10 selten. Von 6 a bis 10 .

8. Drepanocladus exannulatus Gümb.: In 9 sehr selten.

9. Polytrichum strictum Banks.: In 9 sehr selten.

Dr. F. Koppe (Bieleffld) hatte die große Freundlichkeit, die von mir bestimmten Moose nachzuprüen und die Drepanocladus-Arten zu bestimmen, wofür ich ihm herzlich danke.

10. Lycopodium cf. inundatum L.: Eine Spore in Ia Abschnitt 6b.

11. Lycopodium cf. complanatum L.: Eine Spore in Ia 6a.

Perisporlose Farnsporen (im Diagramm unter Filicinae) vereinzelt in Ia $6 \mathrm{c}$, 7, 8, 9, 10, III 7, 8, 9, 10, II 10 ?

12. Botrychium lunaria Sw.: Eine Spore in III 10 und eine in II 10 ?

13. Polypodium vulgare L.: In Ia 7 zwei Sporen und in II 10? eine Spore.

14. Osmunda regalis L.: Sporen vereinzelt in 5, 6a/b, 6c/7, 7, 8, 9 und 10. In TH. III über $60 \%$ in $9 / 10$.

Blattreste in 8/9 selten. - Zu Osmunda gehören vielleicht auch treppenförmige Farntracheiden, die zusammen mit den Blattresten gefunden worden sind. Die Übereinstimmung der Blattreste mit rezentem Vergleichsmaterial ist vollkommen. Die nordamerikanischen Arten Osmunda cinnamomea und claytoniana sind ganz anders.
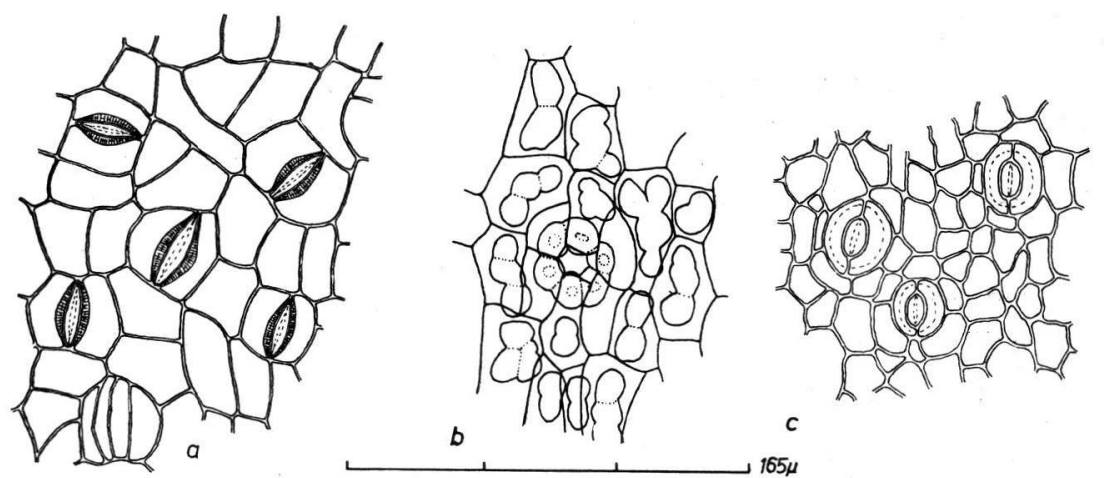

Abb. 4. a) Viscum album, Epidermis; b) Taxus baccata, Epidermis; c) Ilex aquifolium, Epidermis. 
15. Taxus baccata L.: U̇ber 1000 Nadeln: In 6a/b sehr häufig, $6 \mathrm{~b}$ häufig, 6b/c sehr häufig, 6c/7 häufig, 7 sehr häufig, 7/8 sehr selten. - 11 Samen: In $6 a / b$ selten, $6 \mathrm{~b}, 6 \mathrm{~b} / \mathrm{c}$ und $6 \mathrm{c}$ sehr selten. - Holz: In $6 \mathrm{a} / \mathrm{b}$ und $6 \mathrm{~b} / \mathrm{c}$ selten. - $11 \mathrm{Knospen}-$ schuppen: In $6 \mathrm{c}$ sehr selten, in 7 selten. - Mikroskopische Reste also von $6 \mathrm{a}$ bis 7 .

Nadeln: Von den Nadeln existiert meist nur noch die schlauchartige, mit ihren Spaltöffnungsfeldern sehr bezeichnend gebaute Epidermis (Abb. 4b, vergl. Florin 1931), sowie ein unkenntliches, zur Bestimmung nicht mehr brauchbares Leitbündel.

16. Abies alba Mill.: Pollenkörner: Erstes Auftreten gegen Ende von 7 (Ia) bzw. bei Th. I und III etwa in 6c. Ausbreitung gegen Ende von 7. Maximum in 3 (Ia $16 \%$, III $4 \%$, Tн. I $27 \%$, Tн. III $46 \%$, Einzelprobe $51 \%$ ). Abfall gegen Ende von 8 (in Ia fallen 8 und 9 zusammen, in III nicht erfaßt). In TH. I in 9 nur noch etwa $2-3 \%$ bis in 10 . In TH. III noch stärker vertreten, aber zeitlich nicht genau zu erfassen. In I 10a nicht mehr vorhanden. - Über 500 Nadeln: In 7/8 sehr selten, 8 sehr häufig, 8/9 selten, 3 häufig. -- U̇ber 200 Samen: In 7/8 sehr selten, $8 \mathrm{sehr}$ häufig, 8/9 häufig, $9 \mathrm{sehr}$ häufig. - Holz: In 7/8 sehr selten, 8, 8/9 und 9 selten. - Über 100 Fruchtschuppen: In 7/8 selten, 8, 8/9 und 9 häufig.

Unter den Holzresten wurde besonders Wurzelholz gefunden.

17. cf. Larix: Thomson erwähnt aus dem Abschnitt 8 einige ca. $80 \mu$ große, stark gefaltete Pollenkörner, die vielleicht der Gattung Larix angehören können.

18. Picea sp.: Pollenkörner: In Spuren schon in 5 und 6 (zusammenhängend), Anstieg Mitte bis Ende von 7 und 8. Maximum in 9 (Ia 26\%, Tн. I 58\%, Tr. III $59 \%$, Einzelprobe 58\%). In Ia 10 a noch über $10 \%$, in $10 \mathrm{~b}$ unter $10 \%$. TH. I in 10 noch zwischen 15 und $20 \%$. In den allochthonen Schichten in wechselnder Menge. - Kleine Piceapollen, die der Picea omoricoides angehören könnten, wurden richt gefunden. - Holz: In 7/8 sehr selten, 8, 8/9 und 9 selten.

18a. Picea abies (L.) Karsten: Über 300 Nadeln: In $6 \mathrm{~b} / \mathrm{c}$ sehr selten, $6 \mathrm{c}$ selten, $6 \mathrm{c} / 7$ sehr selten, $7 \mathrm{sehr}$ häufig, 7/8 selten, 8 häufig, 8/9 und 9 sehr häufig. Ủber 150 Samen: In $6 c$ sehr selten, 7 häufig, $7 / 8$ und 8 selten, $8 / 9$ häufig, $9 / 10$ und 10 selten. - 87 Knospenschuppen: In $6 \mathrm{c}$ sehr selten, 7 häufig, 7/8 sehr selten, 8, 8/9 und 9 selten, 9/10 sehr selten. - 5 Zapfen: In 9. - Makroskopische Reste also von $6 \mathrm{~b}$ bis 9 .

Auf der Ober- und Unterseite der Nadeln befinden sich je 2 Spaltöffnungsstreifen, die in der Mittelzone je einige Reihen mit in der Längsrichtung gestreckten amphizyklischen Spaltöffnungsapparaten besitzen (Unterschied zu Picea omoricoides vergl. Weber 1898, über die Epidermis vergl. Florin 1931).

Die Länge der fossilen Zapfen beträgt etwa $8-13 \mathrm{~cm}$. Die Zapfenschuppen sind rhombisch vorgezogen und vorne \pm zugespitzt, sie stimmen völlig mit rezenten Schuppen von Picea abies s. str. und nicht mit den vorne abgerundeten Schuppen von $P$. obovata überein.

19. Pinus sp.: Pollenkörner: In Ia in 5 noch über $70 \%$, aber abfallend, in 6 a und $6 \mathrm{~b}$ weiter abfallend. In $6 \mathrm{c}$ und 7 nur noch spurenweise (bzw. in TH. I und III noch zwischen 5 und $10 \%$ in 6c). Anstieg beginnt wieder gegen Ende von 7 bzw. weiter in 8 und 9. In 10 weiterer Anstieg bis auf 80\% (III) bzw. fast 90\% (Ia), Tн. I 73\%, Tн. III nur 21\% (anscheinend nicht erfaßt). Im allochthonen $\mathrm{Ab}-$ schnitt in wechselnder Menge, jedoch stets über 40\%. - Holz: In 8/9, 9 und 9/10 selten. — 28 Nadeln: In 8 und 9 selten. - 10 Samen: In 9 sehr selten, $9 / 10$ selten, 10 sehr selten.

Die Epidermis der Nadeln war fast vollständig zerstört, so daß danach nicht mehr festgestellt werden konnte, ob es sich um Pinus silvestris oder P. mugo 
handelte. Es ist aber wahrscheinlich, daß die Nadeln, die gemeinsam mit den anderen Pinus-Großresten gefunden wurden, von $P$. silvestris stammen.

19a. Pinus silvestris L.: 44 Zapfen: In 8 selten, 8/9 sehr selten, 9 häufig, 9/10 und 10 sehr selten. -

Die Länge der fossilen Kiefernzapfen schwankt zwischen 1,5 und 4,4 cm (Mittelwert: 2,7 cm) bei insgesamt 44 gemessenen Zapfen. Die Schuppen sind relativ schmal, ihre Kanten sind nur schwach aber doch deutlich gerundet.

20. Batrachium sp.: 2 Früchtchen in 9.

21. Thatictrum sp.: Ein Pollenkorn in III $6 \mathrm{~b}$ und ein weiteres in 8 (Einzelprobe).

22. Nymphaea alba L.: 56 Samen: In $6 \mathrm{a} / \mathrm{b}$ sehr selten, $6 \mathrm{~b} / \mathrm{c}$ selten, $6 \mathrm{c}$ und $6 \mathrm{c} / 7$ sehr selten, 7 selten. - Von 6 a bis 7 .

23. Brasenia purpurea Michx.: Etwa 160 gut erhaltene und einige beschädigte, zur Messung unbrauchbare Samen. In 6a/b sehr häufig, 6b sehr selten, $6 \mathrm{~b} / \mathrm{c}$ häufig, $6 \mathrm{c}$ selten. - Von $6 \mathrm{a}$ bis $6 \mathrm{c}$.

Von den Samen besaßen 92 keinen Deckel, 62 hatten einen Deckel, und 4 waren Zweiersamen (deformiert). Für die statistische Auswertung wurden die Samen mit Deckel und ohne Deckel getrennt gemessen. Die Messungen wurden mit einem Meßokular auf 0,05 $\mathrm{mm}$ Genauigkeit durchgeführt; es wurde je eine Messung in der Längsrichtung und eine in der Querrichtung des Samens vorgenommen. Die Längenwerte bei den Samen ohne Deckel schwanken von 2,8 bis 4,0 mm (Mittelwert: 3,31 mm), die Breitenwerte von 2,15-3,1 mm (Mittelwert: $2,67 \mathrm{~mm}$ ). Bei den Samen mit Deckel schwanken die Längenwerte von 2,75-4,0 mm (Mittelwert: 3,44 mm), die Breitenwerte von 2,1-3,05 mm (Mittelwert: 2,65 mm). Für den Deckel kann man also etwa eine Größe von $0,13 \mathrm{~mm}$ annehmen.

H. Kосн hat 1931 umfangreiches rezentes Samenmaterial von Brasenia untersucht, um die von Szafer (1925) versuchte Trennung von Unterarten auf Grund der Größenvariabilität zu prüfen. Es stellte sich aber heraus, daß diese Trennung nicht möglich ist. Für ein größeres japanisches Material von bedeckelten Samen hat H. Koch folgende Werte ermittelt: Länge: 2,9-4,1 mm, Breite: 2,0-3,1 mm. Die mittleren Werte liegen bei KocH für die Länge bei $3,6 \mathrm{~mm}$, für die Breite bei 2,4 mm. Für den Deckel gab Kосн im Mittel 0,14 mm Größe an. Das fossile Material von Wallensen stimmt also recht gut mit dem rezenten japanischen Material von Kосн überein. Die um sehr wenig höheren Breitenwerte der fossilen Samen lassen sich gut durch Deformation bei der Fossilisation erklären. Höckerzellen wurden nur bei sehr wenigen Samen an dem der Mikropyle entgegengesetzten Pol festgestellt (9 Samen des gesamten Materials).

Brasenia purpurea ist in Europa bekanntlich nur in tertiären und interglazialen Ablagerungen mit Sicherheit nachgewiesen worden.

In ihrem heutigen Verbreitungsgebiet (atlantisches Nordamerika, Ostasien, Afrika, Australien) wächst Brasenia hauptsächlich in langsam verlandenden Vereinen (auf tonigen, sandigen, schlammigen Böden), die an eine wenig (ca. $2 \mathrm{~m}$ ) tiefe Zone entlang des Ufers gebunden sind. Brasenia befindet sich im allgemeinen innerhalb der Verlandungszone an offenen Stellen, sog. Seefenstern (Stoller 1908). In Wallensen wurde Brasenia in den Schichten, die offenibar im tieferen Wasser abgesetzt worden waren, niemals gefunden. Sie kam immer in moosreichen Schichten vor (mit Drepanocladus u. a.), die in der Nähe des Ufers gebildet worden waren. 
24. Ceratophyllum demersum L.: 35 Früchte: In 5/6a sehr selten, 6a/b selten, 6b/c häufig, 6c sehr selten. - Von 5--6c, (Zur Bestimmung und Verbreitung vgl. BACKMAN 1943).

25. cf. Ribes: Thомson erwähnt aus dem Abschnitt 8 einige Pollenkörner, die vielleicht der Gattung Ribes angehören können.

26. Rubus idaeus L.: 13 Steinkerne, etwa $2 \mathrm{~mm}$ lang: In 5, 5/6a und 8/9 sehr selten, 9 selten.

27. Rubus fruticosus s. 1. L.: 1 Steinkern von $2,8 \mathrm{~mm}$ Länge in $8 / 9$.

28. Comarum palustre L.: 70 Nüßchen: In $7 / 8$ sehr selten, 8 und $8 / 9$ selten, 9 häufig, $9 / 10$ selten. (Zur Bestimmung vergl. Rudolph 1917.)

29. Sanguisorba officinalis L.: 1 Pollenkorn in III in 10.

30. Epilobium sp.: 1 Pollenkorn in II in 10 ?

31, 32, 33a. Tilia sp.: Pollenkörner: Erstes Auftreten gegen Ende von 5, in $6 \mathrm{a}$ und $6 \mathrm{~b}$ schon in zusammenhängender Kurve, aber noch unter $10 \%$. Anstieg gegen Ende von $6 \mathrm{~b}$. Maximum in $6 \mathrm{c}$ (Ia $29,5 \%$, III $17 \%$, Tн. I $23 \%$, Tн. III $43 \%$, Einzelproben (6c) $68 \%$ und 55,5\%). Abfall gegen Ende von 6c. Zu Beginn von 7 noch über $10 \%$, dann weiter abnehmend aber während 7 noch immer zwischen 0 und 5\%. In 8, 9 und 10 nur noch vereinzelt (in III), in $10 \mathrm{~b}$ nicht mehr.

Versuche, zu einer sicheren Artbestimmung der Pollen zu gelangen, führten zu keinem überzeugenden Ergebnis und sollen daher nicht besprochen werden. Knospenschuppen: In $6 \mathrm{a} / \mathrm{b}$ sehr selten, $6 \mathrm{~b} / \mathrm{c}$ selten, $6 \mathrm{c}$ sehr selten.

31. Tilia cordata MiLl.: Etwa $35 \pm$ gut erhaltene Früchte und einzelne Carpelle. In $6 \mathrm{a} / \mathrm{b}$ selten, $6 \mathrm{~b}$ sehr selten, $6 \mathrm{~b} / \mathrm{c}$ und $6 \mathrm{c}$ selten, 7 sehr selten.

Länge der Früchte einschließlich verbleibendem Griffelrest etwa $5-9 \mathrm{~mm}$. Form verkehrt eiförmig, seltener kugelig, asymmetrisch. Auf dem Endokarp keine Rippen, Carpelle nur durch Nähte verbunden. Abbruchstelle des Fruchtstiels nicht umwallt. Griffelansatz stie]chenartig verlängert, ca. 0,5 mm Durchmesser.

32. Tilia platyphyllos Scop.: Etwa 80 Früchte und einzelne Carpelle (Abb. 5b). In $6 \mathrm{a} / \mathrm{b}, 6 \mathrm{~b}, 6 \mathrm{~b} / \mathrm{c}, 6 \mathrm{c}$ und 7 selten.

Länge einschließlich verbleibendem Griffelrest etwa $6-10 \mathrm{~mm}$. Form kugelig-eiförmig. Größte Breite in der Mitte, seltener im oberen Drittel. Rippen auf dem Endokarp im unteren Teil sehr kräftig, die Abbruchstelle des Fruchtstiels wallartig umwachsend, bis oben deutlich. Griffelansatz kurz, knopfförmig, dick, bis $1 \mathrm{~mm}$ Durchmesser.

Rezente Früchtchen von Tilia intermedia HAYNE zeigen alle Übergänge zwischen T. cordata und platyphyllos. Sie gleichen bald mehr dem einen, bald mehr dem anderen Elter. Auch bei dem fossilen Material fanden sich vereinzelt solche

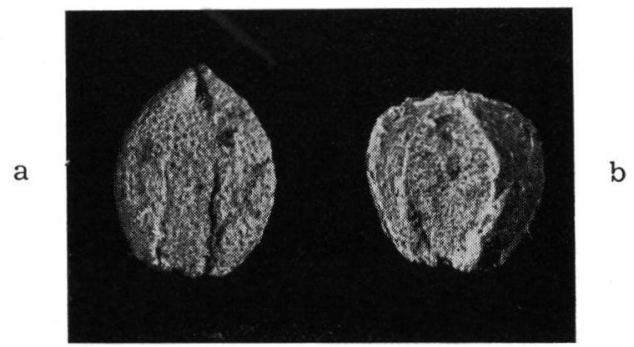

Abb. 5. a) Tilia tomentosa, Früchtchen (2,3x vergr.); b) Tilia platyphyllos, Früchtchen ( $2,3 \mathrm{x}$ vergr.). 
Übergangsformen, die nicht sicher bestimmbar sind und deshalb hier nicht aufgeführt werden sollen.

33. Tilia tomentosa Moench: Etwa 100 Früchte und einzelne Carpelle (Abb. 5a). In $6 \mathrm{a} / \mathrm{b}$ und $6 \mathrm{~b}$ sehr selten, $6 \mathrm{~b} / \mathrm{c}$ selten, $6 \mathrm{c}$ häufig, 7 selten.

Länge etwa $6-10 \mathrm{~mm}$. Form ellipsoidisch, seltener kugelig. Rippen auf dem Endokarp nur im unteren Teil schwach vorhanden, Wall an der Abbruchstelle des Fruchtstiels angedeutet, im oberen Teil nur feine Nähte. Frucht in den Griffelrest allmählich schnabelartig vorgezogen.

BAAs (1931) glaubte in der frühdiluvialen Ablagerung von Schwanheim neben Tilia platyphyllos auch T. tomentosa nachweisen zu können. Seine Abbildungen von fossilen tomentosa- und platyphyllos-Früchten zeigen die erwähnten Unterschiede und stimmen gut mit den Früchten von Wallensen überein. In Rinnersdorf (Stark, Firbas \& Overbeck 1932) wurden ebenfalls Früchte gefunden, die als Tilia platyphyllos vel tomentosa beschrieben wurden. Ich konnte das fossile Material von Rinnersdorf (Sammlung Firbas) mit dem Wallenser Material vergleichen, wobei sich herausstellte, daß die Früchte von Rinnersdorf sämtlich der Tilia tomentosa angehörten. PIECH (1932) zeigt Abbildungen, die als Tilia platyphyllos beschrieben wurden. Sie lassen aber erkennen, daß es sich hierbei auch um Tilia tomentosa handelt (PIECH 1932, Taf. V, Fig. 76). Vielleicht ist $T$. tomentosa auch sonst früher nicht erkannt und zu platyphyllos gerechnet worden, so daß eine Revision älteren Materials noch weitere Funde ergäbe.

Die Beschaffenheit der Oberfläche des Endokarps eignet sich nicht zur Unterscheidung von T. tomentosa und platyphyllos, da die Höcker und Warzen offenbar durch die Fossilisation herausgearbeitet werden.

Heutige Verbreitung (Abb.6): Syrien und Bithynien (und vielleicht westlicher Kaukasus), nord- und nordwestwärts bis Südwestrußland, Rumänien,

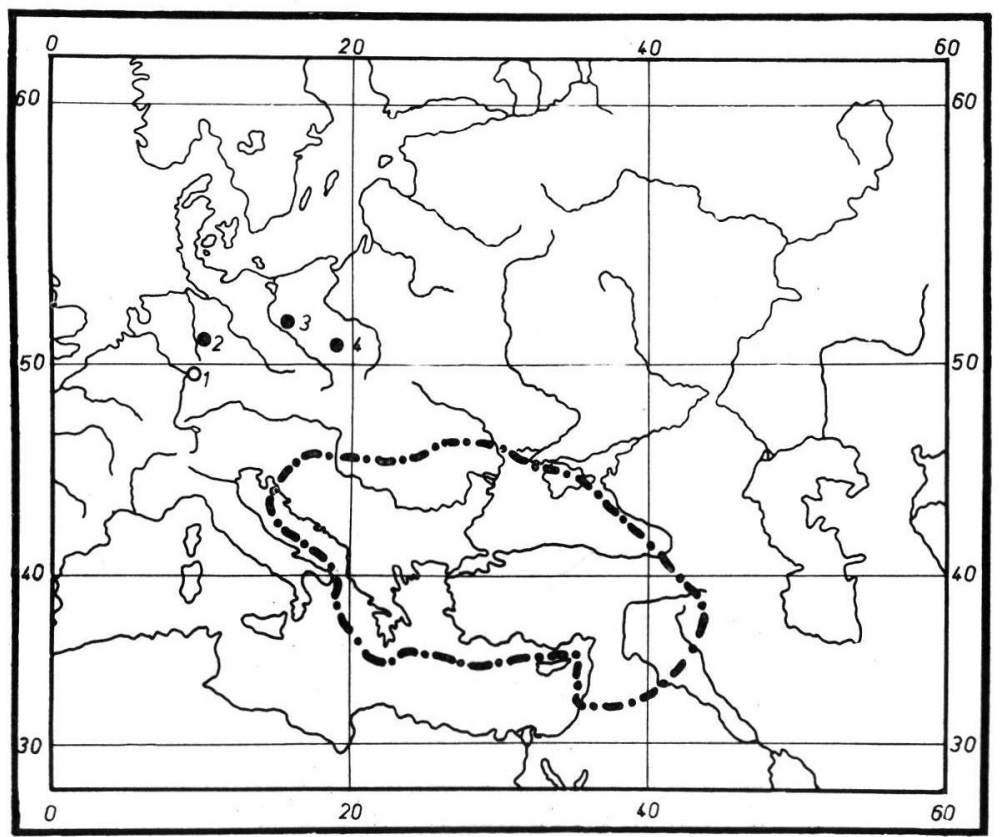

Abb. 6. Heutige Verbreitung von Tilia tomentosa (nach Schmucker 1942).

O frühdiluvialer Fundpunkt; - interglaziale Fundpunkte. 1) Schwanheim,

2) Wallensen, 3) Rinnersdorf, 4) Wielun. 
Siebenbürgen, Süd- und Ostungarn, westwärts bis zum Plattensee, Serbien, Kroatien (Arealkarte bei SCHMUCKer 1942).

34, 35a. Acer sp.: Pollenkörner vereinzelt in $6 a, 6 b, 6 c / 7$ und 7 , sämtlich in gutem Erhaltungszustand.

34. Acer campestre L.: Etwa 70 Früchtchen. In $6 a / b$ häufig, $6 \mathrm{~b}$ sehr selten, 6b/c und $6 \mathrm{c}$ selten, 7 sehr selten. - Von $6 \mathrm{a}-7$. Die Früchte besaßen z. T. noch Reste des Flügels.

35. Acer platanoides L.: 5 Früchtchen, teilweise noch mit Resten des Flügels: In $6 \mathrm{c}$ und 7 sehr selten.

36. Ilex aquifolium L.: Pollenkörner in 5 zwischen 5 und $10^{0} \%$, hier möglicherweise tertiären Ursprungs. In $6 \mathrm{a}, \mathrm{b}, \mathrm{c}$ bis $\mathrm{zu} 5 \%$, in III $6 \mathrm{c}$ über $5 \%$. In 7 vereinzelt noch bis zu 2\%. In 8-10 sehr selten. - 43 Steinkerne: In 6b/c, 6c und 7 selten. - Blattreste (vergl. Abb. 4c): In 6b/c, 6c und 7 selten. Großreste also insgesamt von $6 \mathrm{~b}-7$.

37. Rhamnus frangula L.: Ein Steinkern in 9.

38. Hedera helix L.: Pollenkörner vereinzelt in $6 \mathrm{a}, \mathrm{b}, \mathrm{c}$ und 7 ; in TH. III in $6 \mathrm{c}$ bis $\mathrm{zu} 6 \%$.

Umbelliferae: Pollenkörner in Einzelproben aus $6 \mathrm{~b}$ und $6 \mathrm{c}$ vereinzelt.

39. Quercus sp.: Pollenkörner: Von Beginn der Ablagerung schon in zusammenhängender Kurve. Anstieg gegen Ende von Abschnitt 5 (in Ia) bzw. in 6a/b (III). Maximum in $6 \mathrm{~b}$ (Ia $41 \%$, Th. I $61 \%$, TH. III $63 \%$ ). Abfall gegen Ende von $6 \mathrm{~b}$ bzw. in 6c; in 7 noch zusammenhängend, in wechselnden Verhältnissen schwankend, aber meist unter $10 \%$. In $10 \mathrm{z}$. T. noch vorhanden, aber unter $5^{\circ} \%$. - Etwa $5000 \mathrm{Knospenschuppen:} \mathrm{In} 5$ und 5/6a sehr selten, in $6 \mathrm{a} / \mathrm{b}, 6 \mathrm{~b}, 6 \mathrm{~b} / \mathrm{c}, 6 \mathrm{c}, 7$ und $7 / 8$ sehr häufig, 8 selten, 8/9 sehr selten. - Etwa 40 Cupulae: In 6a/b häufig, 6b sehr selten, $6 \mathrm{~b} / \mathrm{c}$ selten, $6 \mathrm{c}$ und 7 sehr selten. - Eicheln: In $6 \mathrm{a} / \mathrm{b}$ sehr selten, $6 \mathrm{~b} / \mathrm{c}$ selten. - Makroskopische Reste also von 5-9.

Häufig fanden sich Bruchstücke sowie auch ganze Cupulae und Eicheln. Eine Artunterscheidung ist sehr schwierig und unsicher. Eine relativ gut erhaltene Eichel besaß eine fast sitzende, oben lappig verbreiterte Narbe, die für Quercus petraea charakteristisch ist, während bei $Q u$. robur die Narben etwas gestielt und mehr zugespitzt sind (vgl. Kirchner, Loew \& Schröter). $\mathrm{Zu}$ einer sicheren Artdiagnose genügen diese Unterschiede aber noch nicht.

40. Quercus cf. robur L.: 2 relativ gut erhaltene Blätter und Blattreste in $6 c$ und 7 .

Die Blätter von Quercus robur sind im Gegensatz zu Qu. petraea mehr unsymmetrisch und nur sehr kurz gestielt. Auf jeder Seite des Blattes befinden sich 4-5 Lappen, die bei $Q u$. robur stumpflich abgerundet sind, während sie bei Qu. petraea, die beiderseits 5-7 Lappen besitzt, etwas mehr zugespitzt sind. Die Seitennerven verlaufen bei $Q u$. robur im allgemeinen auch in die Buchten, während sie bei $Q u$. petraea in die Lappen und nur am Blattgrund in die Buchten verlaufen (vgl. Gross 1933). Die schwächeren Nerven zwischen den Seitennerven verlaufen bei $Q u$. petraea ziemlich parallel, bei $Q u$. robur ziemlich unregelmäßig. Die Blätter sind bei $Q u$. robur am Grunde geöhrt, während sie bei Qu. petraea keilig in den Stiel verlaufen. Alle diese für $Q u$. robur weitgehend bezeichnenden Merkmale treffen bei dem besonders gut erhaltenen fossilen Blatt $z \mathrm{u}$ bis auf das Fehlen des Blattgrundes.

Es ist möglich, daß in Wallensen beide Eichen-Arten vorgekommen sind.

41. Alnus sp.: Pollenkörner: In 5 mit Werten um $10^{\circ} \%$, in 6 a zunächst noch über $5 \%$, dann weiter abfallend auf unter $5 \%$ in $6 a / b, b$ und $c$ (Ia), bzw. in III 
in $6 \mathrm{a} / \mathrm{b}$ noch zwischen 10 und $20 \%, 6 \mathrm{~b}$ dann wieder zwischen 20 und $25 \%, 6 \mathrm{c}$ unter 5\%. Anstieg gegen Ende von 6c, in Ia weiter ansteigend in 7, in 8/9 Maximum $(26 \%)$, in III $16,5 \%$, in TH. I gegen Ende von $86 \%$, Th. III $21 \%$ (8/9?). Gegen Ende von 9 Abfall bis $10 \mathrm{~b}$ unter $5 \%$ in III, bzw. $0 \%$ in Ia, TH. I $0 \%$, Tн. III unter 5\%. - Holz: In 7/8, 8 und $8 / 9$ sehr selten.

41a. Alnus glutinosa (L.) Gaentner: Über 100 Nüßchen: In 7, 7/8 und 8 häufig, in $8 / 9$ und 9 selten, in $9 / 10$ sehr selten. - Insgesamt also von $7-10$.

Frucht mit \pm gut erhaltenen, nicht durchscheinenden, dicken Flügeln, die also nur undeutlich von der Frucht abgesetzt sind. Bei Alnus incana hebt sich der Flügel dagegen deutlich von der Frucht ab.

42. Betula sp.: Pollenkörner: In 5 und $6 \mathrm{a} / \mathrm{b}$ noch in zusammenhängender Kurve, aber unter $10 \%$ (Ia), bzw. in III von $12-17 \%$, in $6 a ; b$ auf unter $5 \%$ gegen Ende von 6a/b abfallend. In beiden Diagrammen Tномson's in $6 \mathrm{a} / \mathrm{b}$ eben falls unter $5 \%$. In $6 \mathrm{c}$ nur noch vereinzelt auftretend, in 7 vereinzelt bzw. fehlend. Gegen Ende von 7 Anstieg zu höheren Werten in 8 und 9. In 10a Maximum von 20,5\% (Ia), bzw. $45 \%$ (III). In $10 \mathrm{~b}$ wieder Abfall auf unter $10 \%$. In TH. I in 10 weiter ansteigend, aber unter $10 \%$, in TH. III in wechselnden Verhältnissen zwischen 10 und $20^{\circ} \%$ schwankend.

42a. Betula alba L.s. 1.: Etwa 130 Nüßchen ohne Reste des Flügels: In 6b, $6 \mathrm{~b} / \mathrm{c}$ und $6 \mathrm{c}$ sehr selten, 7 selten, 7:8 sehr selten, 8 häufig, $8 / 9$ selten, 9 häufig, 9/10 sehr selten. - 12 Knospenschuppen: In 6a/b, 6b/c, 6c, 8/9, 9 und 9/10 sehr selten. -3 beschädigte und daher nicht näher bestimmbare Fruchtschuppen im Abschnitt 9. - Holzreste: In 7/8, 3, 8/9, 9 und 9/10 sehr selten. - Makroskopische Reste also insgesamt von $6 \mathrm{a}-10$.

43, 44a. Corylus sp.: Pollenkörner in 5 von $7 \%$ auf $80 \%$ ansteigend, in 6 a und $6 \mathrm{~b}$ weiterer Anstieg, Maximuni in $6 \mathrm{~b}$ bei $540 \%$ (Ia), bzw. 915\% (III). In 6c und $\mathrm{zu}$ Beginn von 7 starker Abfall, dann allmählich weiter abfallend. In 8 und 9 unter $10 \%$, in 10 nur noch vereinzelt auftretend.

Auffallend sind die außerordentlich hohen Coryluswerte des Abschnittes 6b, die die Baumpollensumme mehrfach übersteigen (auch Corylus colurna? vgl. 44).

Bekanntlich nehmen die meisten Autoren das Vorhandensein ausgedehnter Haselhaine oder -wälder im frühen Postglazial an (vgl. die nähere Erörterung der Frage bei Firbas 1949). Für die haselreichen interglazialen Abschnitte ist, da die Coryluswerte oft noch höher sind, gleiches erst recht anzunehmen.

Zum Unterschied von dem postglazialen borealen Haselmaximum, das in Nordwestdeutschland, entlang der Ostseeküste, in den westlichen Mittelgebirgen und in einigen südwestlichen Landschaften besonders ausgeprägt ist (vgl. Firbas 1949) und dort deutlich vor das Eichenmischwaldmaximum fällt, ist das inter.glaziale Haselmaximum in Nord- und Nordwestdeutschland, in Dänemark und anderen Landschaften gleichzeitig mit dem Eichenmischwaldmaximum (JEsSEN \& Milthers 1928, Stark, Firbas \& Overbeck 1932, Woldstedt, Rein \& Selle 1951).

43. Corylus cf. avellana L.: Holz in $6 \mathrm{a} / \mathrm{b}$ sehr selten.

Nach Huber \& Rouschal (in Freund 1951) hat das Holz von Corylus colurna in seinen weiteren Gefäßen durchschnittlich weniger und weiter gestellte Leitersprossen als das von C. avellana. (2-5 gegen 5-7). Bei den fossilen Holzresten konnten im Frühholz nur Leitern mit mehr als 5 Sprossen festgestellt werden. Das fossile Holz gehört demnach mit sehr großer Wahrscheinlichkeit zu C. avellana. 
44. Corylus avellana L. vel colurna L.: Etwa 20 z. T. stark beschädigte Nüsse und Bruchstücke von solchen: In $6 \mathrm{a} / \mathrm{b}$ selten, $6 \mathrm{~b}$ sehr selten, $6 \mathrm{~b} / \mathrm{c}$ und $6 \mathrm{c}$ selten, 7 sehr selten.

Form der fossilen Nüsse: Längïich-eichelförmig, größte Breite im oberen Drittel, Spitze ziemlich stumpf. Im oberen Teil stumpf-zweikantig, wohl schon vor der Fossilisierung im Querschnitt ellipsoidisch. (Deshalb wurde zum Vergleich mit rezenten Nüssen nicht die größte Breite sondern der größte Umfang gemessen.) Abbruchstelle \pm stark gewölbt. Oberfläche mit sehr deutlichen Längsrippen, die an der Abbruchstelle mit Zacken ausmünden.

Rezentes Vergleichsmaterial von Corylus colurna von leider unbekanntem Standort stimmte in Form und Beschaffenheit der Oberfläche vorzüglich mit dem fossilen Material überein, war allerdings etwas größer. Maße von 10 fossilen Nüssen: Länge im Mittel 2,2 (2,1-2,35) $\mathrm{cm}$, Umfang im Mittel 4,35 (3,7 bis 4,7$) \mathrm{cm}$. Maße von 20 rezenten Nüssen: Länge im Mittel 2,3 $(2,05-2,65) \mathrm{cm}$, Umfang im Mittel 5,1 (4,4-5,5) cm.

Schneider (1906) gibt für C. colurna eine Breite von $1,2-1,8 \mathrm{~cm}$, eine Länge von $1,7-2 \mathrm{~cm}$ an. Nach Kirchner, Loew \& Schröter ist C. colurna durch eine größere, breitere und stärker gewölbte Abbruchfläche von C. avellana zu unterscheiden; diese Angaben erscheinen aber wenig zutreffend. Die Beschreibung von Willкомм (1887) von C. colurna stimmt vorzüglich mit den rezenten und fossilen Nüssen überein, als Länge gibt er $2 \mathrm{~cm}$ an. Auch Koene (1893) gibt eine ähnliche Beschreibung.

Die Nüsse von C. avellana sind bekanntlich sehr formenreich. GoEschke (1887) beschreibt zahlreiche Wild- und Kulturformen der Hasel und gibt als Maße für die von $C$. avellana abstammenden „Waldnüsse“ an: Länge $12-22 \mathrm{~mm}$, Breite $10-18 \mathrm{~mm}$, Dicke $8-18 \mathrm{~mm}$ (vgl. auch das dort über C. avellana var. pontica Gesagte).

G. Andfrsson (1902) unterscheidet auf Grund umfangreicher Untersuchungen an rezenten und fossilen postglazialen Haselnüssen in Schweden und Finnland ¿ Haupttypen, zwischen denen alle Übergangsformen vorkommen. Das Häufigkeitsverhältnis des runden zu dem langen Haupttypus ist jedoch auffallend konstant bei den fossilen ebenso wie bei den rezenten Nüssen. Die Länge überschreitet $20 \mathrm{~mm}$ nur höchst selten (vgl. weiter das bei Andersson, S. 167 über die Fruchtformen Gesagte). C. \& E. REID (1915) beschreiben fossile Haselnüsse, die stärker gestreift sind als rezente, doch sind hier keine Maße angegeben. MäDLER (1939) gibt für pliozäne Nüsse eine Länge von $1,1-2,0 \mathrm{~cm}$, eine Breite von $0,75-1,85 \mathrm{~cm}$, eine Schalendicke von $0,4-1,5 \mathrm{~mm}$ und $\mathrm{z}$. T. eine kräftige Streifung an.

Die Nüsse der südeuropäischen Corylus maxima MıLL. sind nach Literaturangaben sehr viel länger als breit (Schnemer 2-2,4 cm lang, 1,4-1,5 cm breit, Willкomm: ellipsoidisch, bis $3 \mathrm{~cm}$ lang, Hegr: zusammengedrückt, etwas verlängert, fast walzlich, Maße wie bei Schneider usw.). Sie scheinen der Form nach also von den vorher genannten Arten abzuweichen.

Nach dem vorher Gesagten ist die Übereinstimmung der fossilen Nüsse mit Corylus colurna so gut, daß man mit der Möglichkeit rechnen muß, daß auch diese Art vorgekommen ist. Eine sichere Bestimmung der fossilen Nüsse von Wallensen ist jedoch nicht möglich. Die heutige Verbreitung von C. colurna reicht (nach HEGI) von Südosteuropa nördlich bis Slavonien und zum Banat, östlich bis zum Himalaya. Aus interglazialen Ablagerungen Mittel- und Nordeuropas ist $C$. colurna bis jetzt nicht angegeben worden. 
45. Carpinus betulus L.: Pollenkörner: Erstes Auftreten in 6a (Ia) bzw. in 6c (III). Anstieg gegen Ende von 6c und zu Anfang von 7. Maximum in 7 (Ia: $80 \%$, III: $65 \%$, Th. I: $65 \%$ ). Abfall gegen Ende von 7 und in 8 und 9. In 10 nur noch vereinzelt. -- Etwa 400 Knospenschuppen: In 6c sehr selten, in 7 sehr häufig. - Etwa 640 Nüßchen im Abschnitt 7. - Von 6c bis 7 .

46. Salix sp.: Pollenkörner vereinzelt während der ganzen Ablagerungszeit, stets unter 5\% - 25 Knospenschuppen: In $6 \mathrm{a} / \mathrm{b}$ und $6 \mathrm{~b} / \mathrm{c}$ selten, in 7 sehr selten.

47. Salix aurita L.: Ein kleines, mit rezentem Vergleichsmaterial vorzüglich übereinstimmendes Blättchen im Abschnitt 8 .

48. Salix cf. cinerea L.: Ein Blatt im Abschnitt 6b.

49. Populus sp.: Holzreste in $6 \mathrm{~b}$ und 9 sehr selten.

49a. Populus tremula L.: 120 Knospenschuppen: In $6 \mathrm{a} / \mathrm{b}$ und $6 \mathrm{~b}$ selten, in $6 \mathrm{~b} / \mathrm{c}, 6 \mathrm{c}$ und 7 häufig, $7 / 8$ selten, $8,8 / 9,9$ und $9 / 10 \mathrm{sehr}$ selten. -2 Blätter in 9. - Makroskopische Reste also von 6a bis 10 .

50. Ulmus sp.: Pollenkörner: Schon im Abschnitt 5 vorhanden. In Ia Anstieg auf $31 \%$ in $6 \mathrm{a}$, Abfall auf $22 \%$ in $6 \mathrm{~b}$ und Wieder-Anstieg auf $49 \%$ in $6 \mathrm{c}$. Dann Abfall bis gegen Ende von 7. In 8, 9 und 10 nicht mehr vorhanden. In Profil III in 6 und 7 in wechselnden Verhältnissen schwankend (13-23\%); Maximum in 6c $(26 \%)$, dann Abfall, in 7, 8, 9 nur noch vereinzelt. TH. I: Von 46\% abfallend auf ca. $10 \%$ in $6 \mathrm{c}$; in 7 weiter abfallend, in 8,9 und 10 nur noch vereinzelt. TH. III: In $6 \mathrm{a} / \mathrm{b} 25 \%$, abfallend bis $6 \mathrm{c}$ und $7 / 8$ ?, dann fehlend. In II Abschnitt 10? vereinzelt. - 7 Knospenschuppen: In $6 \mathrm{a} / \mathrm{b}, 6 \mathrm{~b} / \mathrm{c}, 6 \mathrm{c}$ und $7 \mathrm{sehr}$ selten.

Da das Ulmusmaximum in den verschiedenen Diagrammen nicht gleichzeitig ist, muß die Frage, wann die Ulme tatsächlich ihre größte Verbreitung hatte, offen bleiben. Auch Jessen \& Milthers haben in einigen Diagrammen ein sekundäres Ulmusmaximum beobachtet.

51. Urtica dioica L.: Ein Nüßchen in $6 \mathrm{~b} / \mathrm{c}$.

52. Viscum album L.: 1 Pollenkorn in I Abschnitt 7. - Blattreste und Epidermisfetzen (vgl. Abb. 4a): In $6 \mathrm{a} / \mathrm{b}$ sehr selten, $6 \mathrm{~b} / \mathrm{c}, 6 \mathrm{c}$ und 7 selten, $7 / 8,8$, 8/9, 9 und $9 / 10$ sehr selten. - Insgesamt von $6 a$ bis 10 .

Die Blätter und Blattfetzen sind $u$. a. gut an dem charakteristischen Aufbau der Epidermis kenntlich. Die unregelmäßig polygonalen Zellen sind nicht in bestimmter Richtung orientiert. Spaltöffnungen sind auf der Blattunter- und -oberseite vorhanden mit 2 zum Spalt parallel liegenden Nebenzellen. Die Innenwände der Nebenzellen sind stark verdickt, sie bilden einen Wulst über den eingesenkten Schließzellen, die z. T. von den Nebenzellen verdeckt werden. Die Spaltöffnungen liegen \pm senkrecht zur Längsrichtung des Blattes.

Caryophyllaceae: Ein Pollenkorn in II Abschnitt 10?

Chenopodiaceae: Ein Pollenkorn in II Abschnitt 10?

Ericales: Nicht näher bestimmbare Pollenkörner, vereinzelt in den Abschnitten 5 und $6 \mathrm{a} / \mathrm{b}$ in (Ia) und in II Abschnitt 10? Vielleicht tertiären Ursprungs.

53. Empetrum sp.: Pollen in Ia 5 bis zu $3,5 \%$, in 6 a (III) $1 \%$, dann erst wieder im Abschnitt 10.

54. cf. Vaccinium sp.: Pollen in den Abschnitten 8, 9 und 10 vereinzelt, in II 10 ? bis $\mathrm{zu} 8 \%$. In 5 und 6 vereinzelt, hier vielleicht tertiären Ursprungs (daher in der Tabelle nicht verzeichnet).

55. Calluna vulgaris L.: Pollenkörner: In den Abschnitten 6a/b und 7 vereinzelt, in 10 (III) bis zu 12\%. Bei Thomson mit den anderen Ericaceen und 
Sphagnum zusammen in einer Kurve. Nach Tномson spielt unter den Ericaceen Calluna eine große Rolle.

56. Fraxinus excelsior L.: Pollenkörner: In Ia 5 schon in zusammenhängender Kurve, in 6 a bis auf $23 \%$ ansteigend, gegen Ende von 6 a wieder abfallend, dann in $6 \mathrm{~b}$ und $\mathrm{c}$ in zusammenhängender Kurve noch unter 10\%. In 7, 8 und 9 nur noch vereinzelt. In III von Beginn vereinzelt bis zu $5 \%$ in $6 \mathrm{a} / \mathrm{b}$, dann wieder abfallend bis 6c, in 7 fehlend. In den Diagrammen Thomson's nur vereinzelt in 6 , in $\mathrm{I} 6 \mathrm{a} / \mathrm{b}$ bis $4 \%$. -3 beschädigte Flugfrüchte in $6 \mathrm{a} / \mathrm{b}$ und in 7 .

Auffallend sind die hohen Fraxinuswerte in Ia 6a. Mit einer Verwechslung mit Pollenkörnern von Potamogeton ist nicht zu rechnen, da bei allen als Fraxinus bestimmten Pollenkörnern einwandfreie Längsfalten vorlagen.

57. Lycopus europaeus L.: 25 Klausen: In $6 \mathrm{a} / \mathrm{b}$ sehr selten, $6 \mathrm{~b} / \mathrm{c}$ selten, $6 \mathrm{c}$ und 7 sehr selten. - Also von $6 a$ bis 7 .

58. Plantago sp.: In 6b/c (Einzelprobe) ein Pollenkorn.

Rubiaceae: Pollenkörner vereinzelt in 10 (Ia und II) und in Einzelproben aus den Abschnitten $6 \mathrm{~b}$ und $\mathrm{c}$ und 9/10.

59. Sambucus racemosa L.: 6 Steinkerne: In 8,? 8/9 und 9 sehr selten. Ca. $2,5-3 \mathrm{~mm}$ lang und $1-1,5 \mathrm{~mm}$ breit, von Sambucus nigra deutlich durch schwächere Runzelung verschieden.

60. cf. Viburnum sp.: Thомsоn erwähnt Pollenkörner aus dem Abschnitt 8.

61. Valeriana cf. officinalis L.: Ein Pollenkorn in Ia Abschnitt 10.

62. cf. Succisa sp.: Thомson erwähnt ein Pollenkorn aus dem Abschnitt 6a/b.

Compositae: Pollenkörner vereinzelt in 5, 6, 7 und 10.

63. Artemisia sp.: Pollenkörner vereinzelt in 5, 7 und 10 ? (II), aber $2 \%$ nicht überschreitend.

64. Centaurea sect. cyanus L.: 2 Pollenkörner in II 10? eines davon gut erhalten (vgl. Abb. 7a, b).

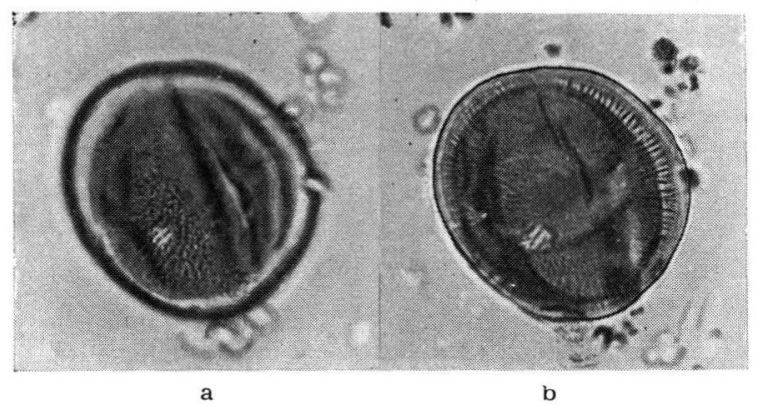

Abb. 7. Centaureu sect. cyanus, Pollen; a) Aufsicht auf die Exine; b) optischer Schnitt ( $6660 \mathrm{x}$ vergr.).
Nach mündlicher Mitteilung von Herrn G. WAGENIrz kommt nach dem Bau der Exine (dicke, verzweigte Stäbchen, außen fast glatt) für Mitteleuropa nur die Sektion cyanus in Frage. Centaurea cyanus selbst scheidet aus, da die Ringfalte nicht von deutlichen costae umgeben ist. Vermutlich handelt es sich um ein -allerdings relativ kleines $(41 \mu)$-Pollenkorn von C. montana oder C. triumfetti.

65, 66, 67a. cf. Potamogeton sp.: Pollenkörner vereinzelt in den Abschnitten bi und 7 .

65. Potamogeton natans L. ${ }^{2}$ ): 16 z. T. beschädigte Steinkerne: In 5, 5/6a und $6 \mathrm{a} / \mathrm{b}$ sehr selten, in $6 \mathrm{~b} / \mathrm{c}$ selten, $6 \mathrm{c}, 8 / 9$ und 9 sehr selten. - Von $5-6 \mathrm{c}$ und in 8 und 9 .

2) Zur Bestimmung dieser und der folgenden Arten vgl. JEssen 1949. 
Länge der unbeschädigten Steinkerne: $2,5-3,2 \mathrm{~mm}$, max. Breite: 2,0-2,2 mm. Steinkerne deutlich gekielt. Die dorsale Klappe reicht bis zur Basis des Griffels, der sich ventral befindet. Auf beiden Seiten des Steinkernes befindet sich eine deutliche zentrale Vertiefung.

66. Potamogeton obtusifolius Mert. et Koch: 55 Steinkerne: In 5 und 5/6a sehr selten, $6 \mathrm{a} / \mathrm{b}$ und $6 \mathrm{~b}$ selten, $6 \mathrm{~b} / \mathrm{c}$ häufig. - Also von $5-6 \mathrm{c}$.

Länge: $1,89-2,73 \mathrm{~mm}$, Breite 1,42-2,2 $\mathrm{mm}$. Ventral- und Dorsalseite konvex, Griffel mittelständig; die dorsale Klappe reicht bis zum Griffel. Klappe schwach gekielt, etwas unregelmäßig. Eine kleine Warze nahe der Basis des Steinkernes laterodorsal auf jeder Seite (Abb. 8a, b).

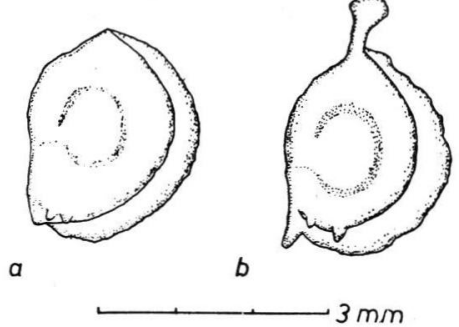

Abb. 8. Potamogeton obtusifolius, Steinkerne.

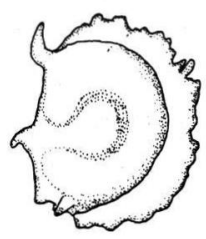

a

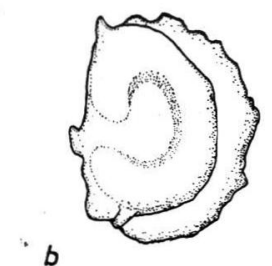

$5 \mathrm{~m} / \mathrm{m}$

Abb. 9. Potamogeton trichoides, Steinkerne.

67. Potamogeton trichoides CHAм, et Schlecht., var. condylocarpus TausCh: 72 Steinkerne: In 5 selten, $5 / 6 \mathrm{a}$ sehr selten, $6 \mathrm{a} / \mathrm{b}$ und $6 \mathrm{~b}$ selten, $6 \mathrm{~b} / \mathrm{c}$ häufig, $6 \mathrm{c}$ sehr selten. - Von 5-6c.

Länge: $2,36-3,04 \mathrm{~mm}$, Breite: $1,73-2,62 \mathrm{~mm}$. Die Ventralseite ist fast gerade, etwas unterhalb der Mitte befindet sich eine kleine Spitze. Die Dorsalseite ist fast halbkreisförmig gekrümmt mit deutlichem, stark höckerigem Kiel; dieser entspringt dicht am Griffel. Basal auf jeder Seite kleine Höckerchen. Am Grunde ein großer Höcker. Die Steinkerne gehören alle der var. condylocarpus an (Abb. $9 \mathrm{a}, \mathrm{b})$. Bei der var. liocarpus ist der Kiel ganzrandig.

68. Najas marina L.: 3 Samen in $6 \mathrm{c} / 7$.

Cyperaceae: Pollenkörner vereinzelt während der ganzen Ablagerungszeit. In Ia 5 bis $9 \%$, dann immer unter $5 \%$ bzw. fehlend. In III $6 \mathrm{a} / \mathrm{b} 10 \%$, sonst immer unter $5 \%$.

69. Eriophorum vaginatum L.: THomson erwähnt aus der allochthonen Serie viel Scheidenepidermen.

70. Carex sect. vignea: 54 Innenfrüchtchen: In $6 \mathrm{~b} / \mathrm{c}$ selten, $6 \mathrm{c}$ und $7 \mathrm{sehr}$ selten, 8 selten, 9 häufig, 9/10 sehr selten, 10 selten. - Also von $6-10$.

71. Carex sect. eucarex: 17 Innenfrüchtchen: In $6 a / b$ selten, $6 \mathrm{~b}, 6 \mathrm{~b} / \mathrm{c}, 6 \mathrm{c}$, $6 \mathrm{c} / 7,8,9$ und 10 sehr selten.

72. Carex pseudocyperus L.: 2 Schläuche in $6 \mathrm{c}$ und in 7 .

73. Carex riparia Curtis: 2 beschädigte Utriculi in $6 \mathrm{~b} / \mathrm{c}$.

Länge: ca. 5,5 und 6,2 $\mathrm{mm}$. Die Schläuche besitzen einen kurzen stark zweizähnigen Schnabel und haben 24-26 Rippen, die abwechselnd stärker und schwächer ausgebildet sind, die randlichen am stärksten. Die Innenfrüchtchen sind dreikantig, ca. $2,7 \mathrm{~mm}$ lang. Von der ähnlichen C. pseudocyperus durch die größere Zahl der Rippen sowie deren schwächere Ausbildung zu unterscheiden. C'. vesicaria besitzt weniger Rippen und einen längeren Schnabel, C. hirta ist langer und behaart. 
74. Dulıchium spathaceum Rich.: 2 Früchtchen in $6 \mathrm{~b} / \mathrm{c}$.

Länge: ca. 3 und $3,5 \mathrm{~mm}$, max. Breite: ca. 0,7 und $1,0 \mathrm{~mm}$, Form länglichoval (vgl. Abb. 10).

Dulichium spathaceum ist ebenso wie Brasenia purpurea aus postglazialen Ablagerungen nicht bekannt. Die Gattung ist heute monotypisch (STOLLER 1909). Im Pliozän sowie in älteren Abschnitten des Diluviums wurde noch eine zweite, heute vollständig ausgestorbene Art dieser Gattung (Dulichium vespiforme Cl. Rein \& El. M. ReID) nachgewiesen. Stoller vermutet, daß die Gattung Dulichium früher, und zwar noch im Diluvium, mehrere Arten aufwies und daß die lebende Art D. spathaceum eine Kollektivart darstellt.

Heute kommt D. spathaceum nur noch im östlichen Nordamerika, von Texas und Florida bis Neufundland, vielfach mit Brasenia purpurea zusammen, vor. Es ist eine Pflanze des Seggen- und Röhrichtgürtels, die ebenso wie Brasenia ein ozeanisches Klima mit viel Niederschlägen liebt.

Gramineae: Pollenkörner in den Abschnitten 5 und $6 a$ zwischen 5 und $10 \%$, sonst immer unter $5 \%$ bzw. fehlend.

75. Phragmites communis Trin.: Rhizomepidermisreste vereinzelt im Abschnitt $6 \mathrm{a} / \mathrm{b}$.

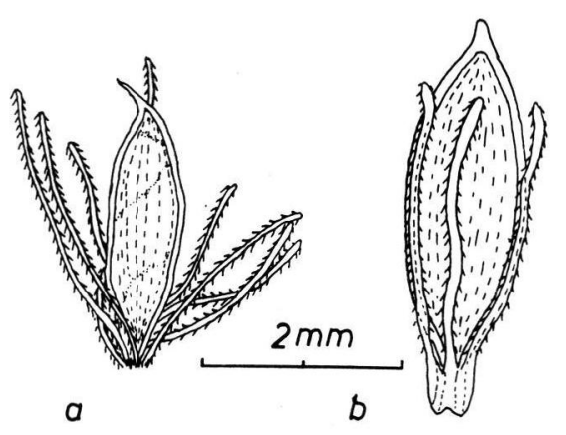

Abb. 10. Dulichium spathaceum, Früchtchen.

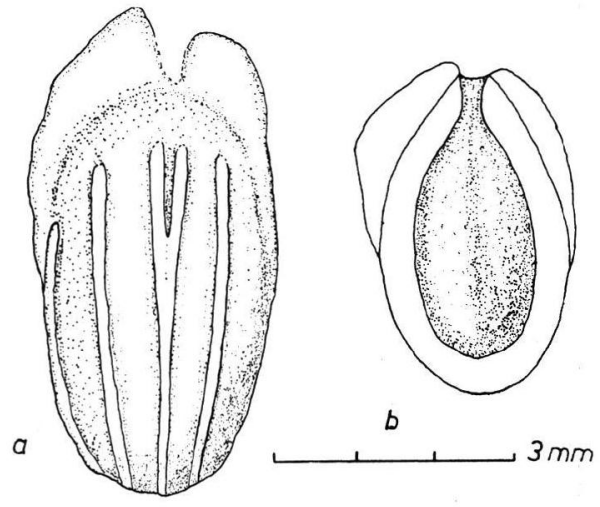

Abb. 11. Unbestimmter Fruchtrest; a) Aufsicht; b) von innen.

76. Sparganium erectum L.: 13 Steinkerne: In $6 \mathrm{a} / \mathrm{b}$ und $6 \mathrm{~b}$ sehr selten, $\mathrm{Eb} / \mathrm{c}$ selten.

77. Sparganium cf. minimum (Hartman) Fries: 7 Steinkerne: In 6b/c selten, in $6 c$ sehr selten.

78. Typha cf. latifolia L.: Pollentetraden vereinzelt während der ganzen Ablagerungszeit.

79. Un besti m m b a re r S a men oder Stein kern: 9 Stück: In $6 \mathrm{~b} / \mathrm{c}, 6 \mathrm{c} / 7$ und 7 sehr selten.

Länge: $3,8-5,4 \mathrm{~mm}$, Breite: 1,9-2,8 mm (Abb. $11 \mathrm{a}, \mathrm{b}$ ). Form länglich-oval, an der oberen (?) Hälfte mit flügelartigem Saum zu beiden Seiten. Oberfläche mit ca. 8 Rippen, von denen die mittleren der Vorder- und Rückseite stärker ausgebildet sind als die übrigen. Die eine Seite ist stärker gewölbt als die ándere. Innen eine ovale Höhlung mit deutlicher Öffnung am oberen und 2 gut zusgebildeten Ecken am unteren Ende. Wandung sklerenchymatisch, die beiden Flügel dagegen aus weichem, schwammigem Gewebe. Eine Bestimmung ist trotz vielfacher Bemühungen nicht gelungen. 
Tabelle 1. Die Vegetationsentwicklung des Interglazials von Wallensen.

\begin{tabular}{|c|c|c|c|c|c|c|c|c|c|c|c|c|c|c|c|}
\hline Zeitabschnitte & 5 & $5 / 6 a$ & $6 a$ & $6 a / b$ & $6 b$ & $6 \mathrm{~b} / \mathrm{c}$ & $6 c$ & $6 c / 7$ & 7 & $7 / 8$ & 8 & $8 / 9$ & 9 & $9 / 10$ & 10 \\
\hline \multicolumn{16}{|c|}{ Vegetation des offenen Wassers: } \\
\hline Fontinalis antipyretica & & & & & & & & & & & & & ss & & ss \\
\hline Drepanocladus fluitans & & & & $\mathrm{h}$ & $\mathrm{h}$ & $\mathrm{h}$ & $\mathrm{h}$ & & $\mathrm{s}$ & & $\mathrm{s}$ & & $\mathbf{s}$ & & $\mathrm{s}$ \\
\hline Drepanocladus exannulatus & & & & & & & & & & & & & ss & & \\
\hline Batrachium sp. & & & & & & & & & & & & & ss & & \\
\hline Nymphaea alba & & & & ss & & $\mathrm{s}$ & SS & ss & $\mathbf{s}$ & & & & & & \\
\hline Brasenia purpurea & & & & $\mathrm{sh}$ & ss & $\mathrm{h}$ & $\mathrm{s}$ & & & & & & & & \\
\hline Ceratophyllum demersum & & ss & & $s$ & & $\mathrm{~h}$ & ss & & & & & & & & \\
\hline Potamogeton natans & ss & SS & & ss & & $\mathrm{s}$ & sS & & & & & ss & ss & ss & \\
\hline Potamogeton obtusifolius & sS & ss & & $\mathrm{s}$ & s & $\mathrm{h}$ & & & & & & & & & \\
\hline Potamogeton trichoides & $\mathrm{s}$ & ss & & $\mathrm{s}$ & $\mathrm{s}$ & $\mathrm{h}$ & ss & & & & & & & & \\
\hline Najas marina & & & & & & & & ss & & & & & & & \\
\hline \multicolumn{16}{|c|}{ Vegetation des Röhricht-und seggengürtels: } \\
\hline Lycopus europaeus & & & & ss & & $\mathrm{s}$ & ss & & ss & & & & & & \\
\hline Carex sp. & & & & $\mathrm{s}$ & ss & $\mathrm{s}$ & $\mathrm{s}$ & SS & ss & & $\mathrm{s}$ & & $\mathrm{h}$ & ss & $\mathrm{s}$ \\
\hline Carex pseudocyperus & & & & & & & & ss & ss & & & & & & \\
\hline Carex riparia & & & & & & ss & & & & & & & & & \\
\hline Dulichium spathaceum & & & & & & ss & & & & & & & & & \\
\hline Phragmites communis & & & & ss & & & & & & & & & & & \\
\hline Sparganium erectum & & & & ss & ss & $\mathrm{s}$ & & & & & & & & & \\
\hline Sparganium cf. minimum & & & & & & $s$ & SS & & & & & & & & \\
\hline Typha cf. latifolia & + & + & + & + & + & + & + & + & + & + & + & + & + & + & + \\
\hline \multicolumn{16}{|c|}{ Vegetation offener Flach- und Hochmoore: } \\
\hline Sphagnum sp. & & & & + & & & & & & ss & $\mathrm{s}$ & $\mathrm{s}$ & $\mathrm{h}$ & $\mathrm{h}$ & $\mathrm{h}$ \\
\hline Aulacomnium palustre & & & & $\mathrm{s}$ & & & & & & & & & & & ss \\
\hline Polytrichum strictum & & & & & & & & & & & & & ss & & \\
\hline Comarum palustre & & & & & & & & & & ss & $\mathrm{s}$ & $\mathrm{s}$ & $\mathrm{h}$ & ss & ss \\
\hline Empetrum sp. & + & + & + & & & & & & & & + & + & + & + & + \\
\hline cf. Vaccinium sp. & & & & & & & & & + & & & + & s & s & $\mathrm{h}$ \\
\hline Calluna vulgaris & & & & & & & & & + & & & + & $\mathrm{S}$ & $s$ & $\mathrm{~h}$ \\
\hline Eriophorum vaginatum & & & & & & & & & & & & & & & $\mathrm{h}$ \\
\hline
\end{tabular}




\begin{tabular}{lllllllllllllllll}
\hline Zeitabschnitte & 5 & $5 / 6 a$ & $6 a$ & $6 a / b$ & $6 b$ & $6 b / c$ & $6 c$ & $6 c / 7$ & 7 & $7 / 8$ & 8 & $8 / 9$ & 9 & $9 / 10$ & 10 & \\
\hline
\end{tabular}

Vegetation der Bruch-und Auewälder und sonstiger Formationen:

Coenococcum geophilum

Leucodon sciuroides

Neckera complanata

Lycopodium cf. inundatum

Lycopodium cf. complanatum

Botrychium lunaria

Polypodium vulgare

Osmunda regalis

Taxus baccata

A.bies alba

Picea $\mathrm{sp}$.

Picea abies

Pinus sp.

Pinus silvestris

Thalictrum sp.

Rubus idaeus

Rubus fruticosus

Sanguisorba officinalis

Epilobium sp.

Tilia sp.

Tilia cordata

Tilia platyphyllos

Tilia tomentosa

Acer sp.

Acer campestre

Acer platanoides

Ilex aquifolium

Rhamnus frangula

Hedera helix

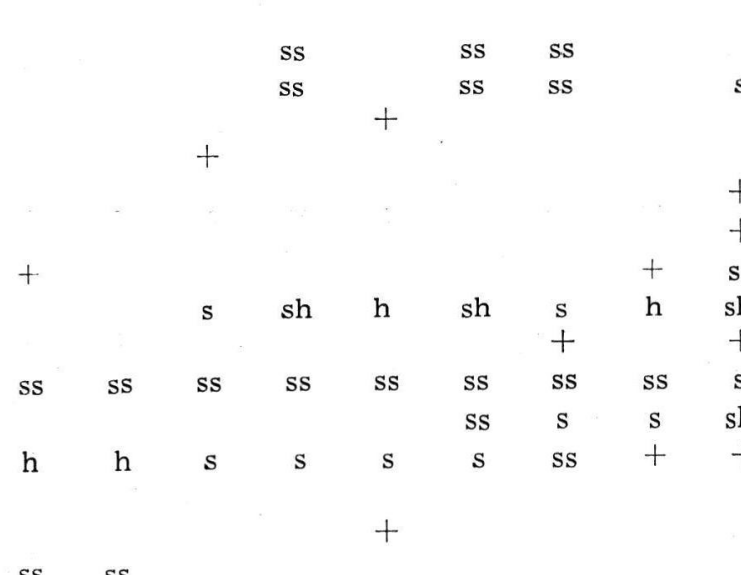

s $\mathrm{h} \quad \mathrm{h}$ sh

Ss Ss

SS
ss
h
s
h
ss

$\mathrm{s} \quad \mathrm{h} \quad \mathrm{h}$

sh sh sh s $\quad$ s

h h h $h$

$h$ sh sh $h$ s

$\begin{array}{lllll}s & \text { s } & h & h & h \\ \text { s } & \text { ss } & h & \text { ss } & \text { ss }\end{array}$

ss $\quad s$

SS

$+$

$+$

$+\mathrm{ss}$ s $\mathrm{s}$ s $\mathrm{s}$ h s s $\mathrm{s}+\mathrm{t}_{+}^{+}+$

$\begin{array}{rrr} & \text { s } & \text { s } \\ & \text { ss } & \text { ss } \\ + & & + \\ & \text { h } & \text { ss }\end{array}$

$\begin{array}{ll}\mathrm{s} & \mathrm{s} \\ \mathrm{s} & \mathrm{h}\end{array}$

$\mathrm{s}$

$\mathrm{s}$
$+\quad+$

ss

s

S

ss ++++

$\begin{array}{lcccccc}\text { s } & \text { s } & \text { s } & \text { h } & \text { h } & \text { s } & \text { s } \\ \text { ss } & \text { ss } & \text { ss } & \text { ss } & \text { ss } & \text { ss } & \text { s }\end{array}$

怘 


\begin{tabular}{|c|c|c|c|c|c|c|c|c|c|c|c|c|c|c|c|}
\hline Zeitabschnitte & 5 & $5 / 6 a$ & $6 \mathrm{a}$ & $6 \mathrm{a} / \mathrm{b}$ & $6 \mathrm{~b}$ & $6 \mathrm{~b} / \mathrm{c}$ & $6 c$ & $6 c / 7$ & 7 & $7 / 8$ & 8 & $8 / 9$ & 9 & $9 / 10$ & 10 \\
\hline Quercus sp. & $\mathrm{s}$ & $\mathrm{s}$ & $\mathrm{s}$ & $\mathrm{sh}$ & $\mathrm{sh}$ & $\mathrm{sh}$ & sh & $\mathrm{s}$ & $\mathrm{sh}$ & $\operatorname{sh}$ & $\mathbf{s}$ & ss & + & + & + \\
\hline Quercus cf. robur & & & & & & & ss & & ss & & & & & & \\
\hline Alnus sp. & $\mathbf{s}$ & $\mathrm{s}$ & ss & ss & Ss & ss & $\mathrm{s}$ & $\mathrm{s}$ & $\mathrm{s}$ & $\mathrm{s}$ & $\mathrm{h}$ & $\mathrm{h}$ & $\mathrm{s}$ & $\mathrm{s}$ & ss \\
\hline Alnus glutinosa & & & & & & & & & $\mathrm{h}$ & $\mathrm{h}$ & $\mathrm{h}$ & $\mathrm{s}$ & $\mathrm{s}$ & ss & \\
\hline Betula sp. & ss & Ss & ss & ss & ss & ss & ss & + & + & $\mathbf{s}$ & $\mathbf{s}$ & $\mathrm{s}$ & $\mathrm{s}$ & $\mathrm{s}$ & $\mathrm{h}$ \\
\hline Betula alba s. 1. & & & & SS & sS & $\mathrm{s}$ & $\mathrm{s}$ & & $\mathrm{s}$ & ss & $\mathrm{h}$ & s & $\mathrm{h}$ & ss & \\
\hline Corylus sp. & $\mathrm{h}$ & $\mathrm{h}$ & $\mathrm{sh}$ & $\mathrm{sh}$ & $\mathrm{sh}$ & $\operatorname{sh}$ & $\operatorname{sh}$ & $\mathrm{h}$ & $\mathrm{h}$ & $\mathrm{s}$ & $\mathrm{s}$ & ss & + & + & + \\
\hline Corylus cf. avellana & & & & ss & & & & & & & & & & & \\
\hline Corylus avellana vel colurna & & & & $s$ & ss & $\mathbf{s}$ & $\mathrm{s}$ & & ss & & & & & & \\
\hline Carpinus betulus & & & + & + & & ss & $\mathrm{s}$ & $\mathrm{h}$ & $\operatorname{sh}$ & $\mathrm{h}$ & $\mathrm{s}$ & ss & ss & + & + \\
\hline Salix sp. & + & + & + & + & + & + & + & + & + & + & + & + & + & + & + \\
\hline Salix aurita & & & & & & & & & & & ss & & & & \\
\hline Salix cf. cinerea & & & & & ss & & & & & & & & & & \\
\hline Populus sp. & & & & & & ss & & & & & & & ss & & \\
\hline Populus tremula & & & & $\mathrm{s}$ & $\mathrm{s}$ & $\mathrm{h}$ & $\mathrm{h}$ & & $\mathrm{h}$ & $\mathbf{s}$ & ss & ss & ss & ss & \\
\hline Ulmus sp. & $\mathrm{s}$ & $\mathrm{s}$ & $\mathrm{h}$ & $\mathrm{h}$ & $\mathrm{h}$ & $\mathrm{h}$ & $\mathrm{h}$ & $\mathrm{s}$ & ss & + & + & + & + & + & $+?$ \\
\hline Urtica dioica & & & & & & ss & & & & & & & & & \\
\hline Viscum album & & & & ss & & $\mathbf{s}$ & $\mathrm{s}$ & & + & ss & ss & ss & ss & ss & \\
\hline Fraxinus excelsior & $\mathbf{s}$ & $\mathbf{s}$ & $\mathrm{h}$ & $\mathrm{h}$ & $\mathrm{s}$ & s & ss & ss & Ss & + & & & & & \\
\hline Plantago sp. & & & & & & + & & & & & & & & & \\
\hline Sambucus racemosa & & & & & & & & & & & ss & ss & ss & & \\
\hline Valeriana cf. officinalis & & & & & & & & & & & & & & & + \\
\hline Artemisia sp. & + & & & & & & & & + & & & & & & $+?$ \\
\hline & 5 & $5 / 6 a$ & $6 a$ & $6 a / b$ & $6 \mathrm{~b}$ & $6 \mathrm{~b} / \mathrm{c}$ & $6 c$ & $6 c / 7$ & 7 & $7 / 8$ & 8 & $8 / 9$ & 9 & $9 / 10$ & 10 \\
\hline
\end{tabular}




\section{Die Vegetationsentwicklung}

In Tabelle 1 sind alle genügend bestimmbaren Pflanzenreste in ihrer Verteilung auf die einzelnen Zeitabschnitte zusammengestellt. Es wurden dabei unterschieden: 1. Pflanzen des offenen Wassers, 2. Pflanzen des Röhrichts, 3. Pflanzen offener Flach- und Hochmoore, 4. Waldpflanzen - angefangen von den Bruchwäldern des Ufers bis zu den Wäldern trockener Mineralböden und Arten anderer Formationen. Verglichen mit anderen Interglazialfloren ist die Zahl der Waldpflanzen verhältnismäßig groß, was mit der Steilheit der Ufer des kleinen, durch Einbruch entstandenen Seebeckens und der Lage der Profile zusammhängen dürfte. An Hand dieser Tabelle lassen sich nun die nach den Pollendiagrammen unterschiedenen Waldperioden zusammenfassend kennzeichnen ${ }^{3}$ ).

5. Kiefern-Eichenmischwaldzeit: Der Abschnitt ist in den untersuchten Profilen nur schlecht erfaßt. Wenigstens zu Beginn dürfte Pinus noch eine große Rolle gespielt haben. Quercus,Fraxinus, Ulmus und Corylus sind in kräftiger Ausbreitung begriffen, und von Quercus liegen auch schon Funde von Knospenschuppen vor. Alnus, Betula, Tilia und Picea sind nur mit geringen Pollenwerten vertreten, die nicht unbedingt auf ein Vorkommen im Gebiet zurückgehen müssen. Von der sonstigen Flora wurde nur wenig gefunden. Erwähnenswert ist Potamogeton trichoides (neben P. natans und obtusifolius) als Anzeichen der beginnenden Ausbreitung der wärmeliebenden Wasserflora, die den nächsten Abschnitt auszeichnet, sowie der Nachweis von Osmunda, die während der Eichenmischwaldzeit fehlt und erst wieder mit Beginn der Hainbuchenzeit auftritt. (Sediment noch sehr minerogen.)

6. Hasel-Eichenmischwaldzeit: Nach dem Pollendiagramm beherrschen Eichenmischwälder mit Corylus, Ulmus, Fraxinus, Quercus und besonders im letzten Unterabschnitt (6c) auch mit Tilia die Umgebung. Reiche Funde von Großresten bestätigen und erweitern diese Befunde durch den Nachweis von Corylus avellana vel colurna, Quercus sp. bzw. Q. robur, Ulmus sp., T'ilia tomentosa (ziemlich häufig), T. platyphyllos (weniger häufig), T. cordata (relativ selten), außerdem Acer campestre und platanoides, Populus tremula, Betula alba (selten), Salix sp. und cf. cinerea. Von Coniferen ist Taxus baccata häufig. Wichtig sind auch die Funde von Hedera helix, Viscum album (in 6c) und Ilex aquifolium. Im letzten Abschnitt ist die herannahende Ausbreitung von Carpinus und Picea durch die ersten Großreste dieser Arten angedeutet.

Der reichen Zusammensetzung der Gehölze entspricht eine reiche, wärmeliebende Wasser- und Ufervegetation mit Nymphaea alba, Brasenia purpurea (zusammen mit Drepanocladus fluitans), Ceratophyllum demersum, Potamogeton natans, P. obtusifolius, P. trichoides als Wasserpflanzen und Lycopus europaeus, Carex riparia, Dulichium spathaceum, Phragmites communis, Sparganium erectum als Arten des Röhrichts. Von der Wasserflora sind die Interglazialpflanzen Brasenia und Dulichium auf die Hasel-Eichenmischwaldzeit beschränkt.

7. Hainbuchenzeit: Nach dem Pollendiagramm herrscht Carpinus deutlich vor, daneben sind Corylus, Tilia, Quercus und Alnus nicht selten, auBerdem Fraxinus, Ulmus und Picea (diese in beginnender Ausbreitung) vorhanden. Gegen Ende erscheint Abies. Pollen von Pinus und Betula fehlt fast völlig.

3) Es ist zu beachten, daß die ältesten frühinterglazialen Abschnitte 1-4, die wir im Vergleich mit anderen Fundstellen zu erwarten haben, hier nicht erfaßt worden sind. 
Die Funde von Großresten bestätigen die große Häufigkeit der Hainbuche in der näheren Umgebung. Sie zeigen außerdem, daß sich die Gehölze der Eichenmischwaldzeit noch erhalten haben. So sind durch Großreste von den Gliedern des Eichenmischwaldes Quercus (reichlich, wohl Q. robur), Tilia platyphyllos, T. tomentosa und T. cordata, Ulmus sp., Acer campestre und A. platanoides sowie Corylus colurna vel avellana nachgewiesen. Taxus ist ebenfalls noch reichlich vorhanden und stirbt dann gegen Ende des Abschnittes anscheinend aus. Vorkommen in Seenähe sind außerdem für Populus tremula, Betula alba, Picea abies (schon sehr reichlich) und, erst von diesem Abschnitt an, für Alnus glutinosa erwiesen. Auch Ilex, Hedera und Viscum hat die Hainbuchenzeit mit der Eichenmischwaldzeit noch gemein.

Die Wasser- und Ufervegetation ist gegenüber der Eichenmischwaldzeit auffällig verarmt. Nur Nymphaea und Lycopus sind von den früher erwähnten Arten noch vorhanden und nur an der Wende 6/7 kommen Najas marina und Carex pseudocyperus (sehr selten) neu hinzu. Außerdem finden sich seit der Wende 6/7 regelmäßig Sporen von Osmunda.

Bei den folgenden Abschnitten ist zu bedenken, daß von ihnen weniger Material durchgesehen worden ist als aus der Eichenmischwald- und Hainbuchenzeit.

8. Tannenzeit: Aus dem Pollendiagramm muß man eine beträchtliche Häufigkeit von Abies und (örtlich) von Alnus, einen starken Rückgang der thermisch anspruchsvolleren Arten einschließlich der Hainbuche und eine immer stärker werdende Ausbreitung von Picea folgern.

Die Großreste bestätigen die große Häufigkeit von Abies alba, Picea abies und Alnus glutinosa. Vom Eichenmischwald wurde nur noch Quercus (sehr seltene Knospenschuppen) gefunden. Vom Uferrand stammen wohl Salix aurita und Populus tremula. Pollen von Hedera ist nicht mehr vorhanden, solcher von Ilex selten (Großreste fehlen). Viscum ist hingegen durch Blattfunde erwiesen.

Die Wasserflora ist schlecht erfaßt, nur Potamogeton natans ist von der Wende $8 / 9$ bekannt. Osmunda wird häufiger. Von Rubus idaeus, R. fruticosus und Sambucus racemosa wurden an der Wende 8/9 Steinkerne nachgewiesen.

Sehr interessant ist das Auftreten einer Pflanzengruppe, die sich nun offenbar im Zusammenhang mit einer beginnenden meso- bis oligotrophen Vermoorung einfindet oder häufiger wird. Man kann dazu Pinus silvestris (Zapfen, wohl auch zu dieser Art gehörige Nadeln), Betula alba, Comarum palustre und cf. Vaccinium zählen und das häufigere Vorkommen von Sphagnumsporen so deuten.

9. Fichtenzeit: Im Pollengehalt herrscht Picea vor, die Pollen aller übrigen Bäume werden immer spärlicher mit Ausnahme von Pinus und Betula, die zunehmen.

Auch das bestätigen die Großreste, unter denen Picea und - wohl mehr aus dem älteren Teil des Abschnittes - auch noch Abies besonders häufig sind. Von Alnus glutinosa wurden noch einige Früchte gefunden; viel häufiger sind aber nun die Großreste von Pinus silvestris und Betula. Auch die oligotrophe Moorkomponente ist bereichert: Zu Sphagnum, von dem jetzt auch Blätter gefunden sind, ist Polytrichum strictum, zu Comarum und cf. Vaccinium regelmäßiger Calluna (Pollen) getreten. Rhamnus frangula ist weiter zu erwähnen, ebenso die häufigen Osmunda-Sporen, Sambucus racemosa und immer noch Viscum. In der Wasserflora tritt neben Potamogeton natans und einigen Moosen (Drepanocladus fluitans, Drepanocladus exannulatus, Fontinalis antipyretica) Batrachium auf. 
10. K i ef e r n z e it: Nach dem Pollendiagramm sind Pinus (vorherrschend), Betula und Picea häufig, alle anderen Holzarten nur durch geringe Werte vertreten, die nichts über ihr Vorkommen im näheren Gebiet besagen. Auch unter clen Großresten sind nur Pinus silvestris, Picea abies und (an der Wende 9/10) Betula alba festgestellt worden. Zu einer spärlichen Wasserflora mit Fontinalis und Drepanocladus fluitans kommen Reste einer Hochmoorflora mit Sphagnum, Aulacomnium palustre, Eriophorum vaginatum, Empetrum, cf. Vaccinium und Calluna. Osmunda-Sporen sind ziemlich selten geworden. Bemerkenswert sind Pollen von Sanguisorba officinalis und Valeriana cf. officinalis.

Versucht man die nachgewiesenen Perioden $\mathrm{klimatis} \mathrm{ch}$ zu deuten, so kann kein Zweifel darüber bestehen, daß die Tannen-Fichten- und Kiefernzeit kühler war als die Eichenmischwald- und Hainbuchenzeit, wobei wir zumindest während der Fichten- und Kiefernzeit sowohl mit niedrigeren Sommertemperaturen wie mit einer kürzeren Vegetationsperiode zu rechnen haben. In der Abfolge dieser 3 Abschnitte spiegelt sich offenbar die Klimaverschlechterung der herannahenden neuen Eiszeit wieder. Ansätze zur Hochmoorbildung oder eine solche selbst, die starke Zunahme der Calluna- und Sphagnum-Werte deuten darauf, daß die Niederschläge wesentlich höher waren als in den spätglazialen Birken-Kiefernzeiten (vgl. Firbas 1951).

Die Frage, ob das Temperatur-Optimum des Interglazials in der Eichenmischwaldzeit (6) oder der Hainbuchenzeit (7) oder gar schon in der KiefernEichenmischwaldzeit (5) lag, läßt sich an Hand der Waldentwicklung weniger sicher entscheiden, da man nicht weiß, ob die Ausbreitung von Carpinus nicht nur mit einer entsprechend späten Zuwanderug zu erklären ist. Doch wird die wahrscheinlichere Annahme, daß die Sommer während der Eichenmischwaldzeit am wärmsten waren, dadurch gestützt, daß die Wasserflora während dieses Abschnittes am reichsten war und Brasenia, Dulichium und Potamogeton trichoides fast nur in diesem Abschnitt gefunden wurden.

Von Pflanzen, die einen Hinweis auf die thermische Ozeanität geben können, ist Ilex durch sehr wahrscheinlich hierher gehörige Pollen zu allen Zeiten, durch Großreste aber nur im jüngeren Teil der Eichenmischwald- und in der Hainbuchenzeit vertreten, Taxus während des größten Teils der Eichenmischwaldund Hainbuchenzeit, Hedera ebenso während beider Perioden, Osmunda einmal (in Spuren) während der Kiefern-Eichenmischwaldzeit (5) und dann erst wieder vom Ende der Eichenmischwaldzeit bis in die Kiefernzeit (10). Auch das völlige Fehlen von Pinus während der Hainbuchenzeit spricht - als Ausdruck eines ausgeprägten Laubholzgebietes - für relativ milde Winter. Gleichzeitig ist aber der subkontinentale Acer platanoides, dessen Nordwestgrenze (von Belgien und der Eifel kommend) heute durch das Weserbergland zieht, gegen Ende der Eichenmischwaldzeit und in der Hainbuchenzeit nachgewiesen. Danach dürfte das Gebiet von Wallensen während eines großen Teils des letzten Interglazials ähnlich wie heute einen gewissen $\mathrm{subozeanischen} \mathrm{Klima-}$ charakter besessen haben, besonders während der Eichenmischwaldzeit, vielleicht auch noch während der Hainbuchenzeit; wahrscheinlich war es jedoch wesentlich wärmer als heute. Nach der heutigen Nordgrenze von Tilia tomentosa (vgl. Abb.6) beurteilt, müßte die Julitemperatur während $6 \mathrm{c}$ und 7 mindestens $20-21^{\circ}$ betragen haben, während sie heute bei $17-18^{\circ}$ liegt ${ }^{4}$ ). Gleichzeitig cürrten nach dem Ilex-Vorkommen und dem Kiefern-Rückgang die Januar-

$\left.{ }^{4}\right)$ Ist die Bestimmung von Corylus colurna richtig, dann würde das die durch Tilia tomentosa wahrscheinlich gemachten Klimaverhältnisse bestätigen bzw. bekräftigen. 
mittel kaum unter 0 bis $-1^{\circ}$ gelegen haben. Schon an der Wende der Tannenzur Fichtenzeit aber dürften Junimittel von $13-14^{\circ}$ unterschritten worden sein und die starke Ausbreitung der Fichte könnte außerdem mit einer Zunahme der Winterkälte zusammenhängen. Doch soll nicht weiter versucht werden, den interglazialen Klimaverlauf abzuleiten, da dies besser auf Grund des Vergleichs einer großen Zahl von Fundstellen gleichen Alters geschieht.

Wie schon eingangs erwähnt worden ist, lassen sich die Waldperioden des Interglazials von Wallensen, soweit sie erfaßt werden konnten, ohne Schwierigkeit der Gliederung des letzten Interglazials zuordnen, wie sie in Dänemark JESSEN \& Milthers (1928), im nordwestdeutschen Flachland zu einem guten Teil schon C. A. Weber (seit 1890), in jüngster Zeit Woldstedt, Rein \& Selle (1951) erkannt haben. Als bezeichnende Züge dieses Interglazials gelten: eine verspätete Ausbreitung von Corylus gegenüber dem Eichenmischwald, ein während der Eichenmischwald- und Hainbuchenzeit nur sehr geringer Anteil von Picea und das völlige Fehlen von Fagus (zumindest im nordwestlichen Mitteleuropa). Die beiden letztgenannten Merkmale gelten auch für Wallensen - von Fagus wurde kein einziges Pollenkorn gefunden. Ob sich die Hasel in Wallensen nach dem Eichenmischwald ausgebreitet hat, läßt sich nicht sagen, da die Diagramme nicht weit genug zurückreichen.

Ein Vergleich der Diagramme mit anderen Fundstellen soll im übrigen nicht vorgenommen werden. Hingewiesen sei nur darauf, daß in Wallensen die AlnusWerte relativ niedrig sind, was sich wohl aus der Form und Lage des kleinen Einsturzbeckens, dem Fehlen größerer randlicher Bruchmoorgebiete, erklärt. Ferner darauf, daß die offensichtlich von Ost nach West gerichtete Ausbreitung ảer Tanne insoweit bestätigt wird, als Abies in Wallensen erst gegen Ende der Carpinuszeit auftritt, während sie in Rinnersdorf schon vor dem CarpinusMaximum, weiter östlich noch früher erscheint (vgl. Finbas 1951). Jedenfalls kann die Waldentwicklung, wie sie bisher nur im Flachland nördlich der Mittelgebirge als für das letzte Interglazial bezeichnend erkannt worden ist, durch Wallensen auch für den Bereich der nordwestlichen Mittelgebirge als gültig angesehen werden.

\section{$\mathrm{Zusammenfassung}$}

1. Von dem zuerst 1907 von Menzel beschriebenen, vor kurzem (1951) auch von P. W. Tномson pollenanalytisch untersuchten Interglazial von Wallensen wurden noch 3 weitere Diagramme aufgestellt, ein Profil und über 50 Einzelproben auf Großreste untersucht. Die nachgewiesene Folge der Waldperioden: 5. Kiefern-Eichenmischwaldzeit - 6. Hasel-Eichenmischwaldzeit - 7. Hainbuchenzeit - 8. Tannenzeit - 9. Fichtenzeit - 10. Kiefernzeit, stimmt - mutatis mutandis - völlig mit der Periodenfolge überein, die in Dänemark und im nordwestdeutschen Flachland als für das letzte Interglazial bezeichnend erkannt worden ist. (Die ältesten frühinterglazialen $\mathrm{Ab}-$ schnitte sind nicht erfaßt worden). Danach kann (mit Thomson) Wallensen dem letzten Interglazial zugeordnet und die erwähnte Waldfolge auch für die nordwestlichen Mittelgebirge als gültig angesehen werden. Von Fagus wurde kein einziges Pollenkorn nachgewiesen.

2. Die pollenanalytischen Befunde konnten durch die Untersuchung von Großresten, u. a. durch zahlreiche Funde von Knospenschuppen ergänzt und erweitert werden. Insgesamt wurden 80 Sippen, meist Arten, z. T. Gattungen oder Familien, bestimmt, wonach Wallensen als artenreiche Interglazialflora bezeichnet werden kann. Die Wasserflora war während der Hasel-Eichenmischwaldzeit am reichhaltigsten; für diese Zeit konnten auch die in Europa heute ausgestorbenen interglazialen Leitpflanzen Brasenia purpurea und Dulichium spathaceum nachgewiesen werden. Bemerkenswert ist auch der Nachweis der heute südosteuropäischen Tilia tomentosa. Ein merkwürdiger Fruchtrest (Steinkern?), der unter Nr. 79 beschrieben worden ist und 
wahrscheinlich von keiner mitteleuropäischen Art stammt, gehört sowohl der HaselEichenmischwald- wie der Hainbuchenzeit an.

3. Reiche Funde von Ilex, Hedera, Taxus und Osmunda bezeugen eine gewisse thermische Ozeanität des Gebiets während aller Zeiten. Während der Hasel-Eichenmischwaldzeit und der Hainbuchenzeit dürfte das Gebiet aber sommerwärmer gewesen sein als heute.

\section{Literaturverzeichnis}

Andersson, G.: Hasseln i Sverige fordom och nu. - Sv. Geol. Unders. Ser. Ca No. 3, 1902.

BaAs, J.: Eine frühdiluviale Flora im Mainzer Becken. - Z. f. Bot. 25, 1932.

BACKMan, A. L.: Ceratophyllum submersum in Nordeuropa während der Postglazialzeit. - Acta Bot. Fenn. 31, 1943.

Fırbas, F.: Waldgeschichte Mitteleuropas I, 1949. - Die quartäre Vegetationsentwicklung zwischen den Alpen und der Nord- und Ostsee. - Erdkunde 5, 1951.

Florin, R.: Untersuchungen zur Stammesgeschichte der Coniferales und Cordaitales I. Morphologie und Epidermisstruktur der Assimilationsorgane bei den rezenten Coniferen. - Kgl. Svenska Vetenskapsak. Handlingar. 3. Ser. 10/1, 1931.

Fratschner, W.-Th.: Facies und Tektonik im Hils. - Diss. Clausthal 1950.

Freund, H.: Handbuch der Mikroskopie in der Technik, Bd. V, Teil I, 1951.

Goeschke, F.: Die Haselnuß, ihre Arten und ihre Kultur, 1887.

Gross, H.: Die Traubeneiche in Ostpreußen. - Z. f. Forst- u. Jagdwesen 65, 1933.

HEGI, G.: Flora von Miteleuropa. München $1906 \mathrm{ff}$.

Hesmer, H.: Samen- und Knospenschuppenanalysen in Mooren. - Z. f. Forst- u. Jagdwesen, 67, 1935.

Hoffmeister, I. \& Schnelle, F.: Klimaatlas für Niedersachsen, 1945.

Jessen, K.: Studies in Late Quaternary deposits and Flora-History of Ireland. - Proc. Roy. - Irish Academy 52, Sect. B, Nr. 6, 1949.

Jessen, K. \& Milthers, V.: Stratigraphical and paleontological Studies of Interglazial Fresh-water Deposits in Jutland and Northwest Germany. - Danm. Geol. Undersög. II. Raekke, Nr. 48, 1928.

v. Kirchner, O., Loew, E. \& Schröter, C.: Lebensgeschichte der Blütenpflanzen Mitteleuropas. Stuttgart $1908 \mathrm{ff}$.

Koene, E.: Deutsche Dendrologie, 1893.

Косн, Н.: Zur Gliederung der Gattung Brasenia auf Grund der Samenvariabilität. Senckenbergiana 13, 1931.

MädLER, K.: Die pliozäne Flora von Frankfurt am Main. - Abh. Senck. naturf. Ges. 446, 1939.

Menzel, H.: Über eine diluviale Süßwasser- und Torfablagerung bei Wallensen im südlichen Hannover. - Z. deutsch. geol. Ges. 54, Sber. v. 3. Dez. 1902. Beiträge zur Kenntnis der Quartärbildungen im südlichen Hannover. Die Interglazialschichten von Wallensen in der Hilsmulde. - Jb. preuß. geol. L.-A. 24, 1907.

PIEch, K.: Das Interglazial in Szczercow (östlich von Wielun, Wojewodschaft Lodz). Ann. soc. géol. Pol. 8, I, 1932.

Rabien, I.: Zur Bestimmung fossiler Knospenschuppen. - Paläont. Z. 27, 1953.

REID, C. \& E.: The Pliocene Flora of the Dutch-Prussian Border. - Mededel. Rijksopsp. Delfsstoffen, Nr. 6, 1915.

Rink, A.: Die Ith-Hilsmulde. - Hann. geogr. Arb. H. 1, 1942,

RudolPH, K.: Untersuchungen über den Aufbau böhmischer Moore. I. Aufbau und Entwicklungsgeschichte südböhmischer Hochmoore. - Abh. d. k. u. k. zool. bot. Ges, 9, 1917.

Schmucker, Th.: Die Baumarten der nördlich. gemäßigten Zone und ihre Verbreitung. Silvae Orbis. Schriftenreihe der Int. Forstzentrale, Nr. 4, 1942.

Schneider, C. K.: Illustriertes Handbuch der Laubholzkunde, Bd. I, 1905.

Selle, W.: Die Interstadiale der Weichselvereisung. - Eiszeitalter u. Gegenwart 2, 1952.

Stark, P., Firbas F. \& Overbeck, F.: Die Vegetationsentwicklung des Interglazials von Rinnersdorf in der östl. Mark Brandenburg. - Abh. nat. Ver. Bremen 1932. 
Stoller, J.: Über die Zeit des Aussterbens der Brasenia purpurea Michx. in Europa, speziell Mitteleuropa. - Jb. preuß. geol. L.-A. 29, 1908. - Über das fossile Vorkommen der Gattung Dulichium in Europa. - Jb. pr. geol. L.-A. 30, I, 1909.

SzAFER, W.: Zur Frage der Vielgestaltigkeit, Herkunft, sowie des Aussterbens von Brasenia purpurea im europäischen Diluvium. - Veröff. geobot. Inst. Rübel, Schröterfestschrift, 1925.

Thomson, P. W.: Das Interglazial von Wallensen im Hils. - Eiszeitalter u. Gegenwart 1, 1951

Weber, C. A.: Úber eine omoricaartige Fichte aus einer dem älteren Quartär Sachsens angehörenden Moorbildung. - Engl. bot. Jahrb. 24, 1898.

WiLLкомм, M.: Forstliche Flora von Deutschland und Österreich, 1887.

WoldstedT, P.: Ưber die stratigraphische Stellung einiger wichtiger Interglazialbildungen. - Z. deutsch. geol. Ges. 99 (1947). 1949.

Woldstedt, P., Rein, U. \& Selle, W.: Untersuchungen an nordwestdeutschen Interglazialen. - Eiszeitalter u. Gegenwart 1, 1951

Ms. eingeg. 21. 10. 52.

Anschr. d. Verf.: Dr. Ilse Rabien, Göttingen, Birkenweg 22. 